\title{
COOL COMPANIONS TO WHITE DWARF STARS FROM THE TWO MICRON ALL SKY SURVEY ALL SKY DATA RELEASE
}

\author{
D. W. Hoard, S. Wachter, Laura K. Sturch, ${ }^{1}$ Allison M. Widhalm, ${ }^{2,3}$ Kevin P. Weiler, ${ }^{4,5}$ \\ Magaretha L. Pretorius, ${ }^{6}$ Joseph W. Wellhouse, ${ }^{1,3}$ and Maxsim Gibiansky ${ }^{1}$ \\ Spitzer Science Center, California Institute of Technology, Pasadena, CA 91125, USA; hoard@ipac.caltech.edu, wachter@ipac.caltech.edu \\ Received 2007 February 2; accepted 2007 February 28
}

\begin{abstract}
We present the culmination of our near-infrared survey of the optically spectroscopically identified white dwarf stars from the McCook and Sion catalog, conducted using photometric data from the Two Micron All Sky Survey final All Sky Data Release. The color selection technique, which identifies candidate binaries containing a white dwarf and a low-mass stellar (or substellar) companion via their distinctive locus in the near-infrared color-color diagram, is demonstrated to be simple to apply and to yield candidates with a high rate of subsequent confirmation. We recover 105 confirmed binaries, and identify 27 firm candidates (19 of which are new to this work) and 21 tentative candidates ( 17 of which are new to this work) from the 2MASS data. Only a small number of candidates from our survey have likely companion spectral types later than M5, none of which is an obvious L-type (i.e., potential brown dwarf) companion. Only one previously known white dwarf + brown dwarf binary is detected. This result is discussed in the context of the 2MASS detection limits, as well as other recent observational surveys that suggest a very low rate of formation (or survival) for binary stars with extreme mass ratios.
\end{abstract}

Key words: binaries: general — infrared: stars — stars: low-mass, brown dwarfs — white dwarfs

Online material: color figures, machine-readable table

\section{INTRODUCTION}

White dwarf (WD) stars play key roles in a wide variety of astrophysically important scenarios. They not only represent the distant future of our own Sun, but are the endpoints in the evolution of the majority of stars in the Galaxy. As the relic cores of normal stars, WDs reveal the outcome of stellar evolution processes, and expose material created during a stellar lifetime of nuclear burning to direct examination. Yet one of the most intriguing aspects of the observational study of WDs results simply from their role in facilitating the discovery of another type of object.

Detecting low-mass stellar (or substellar) companions to WDs offers many advantages compared to main-sequence primaries. In the latter case, faint low-mass companions are often hidden in the glare of the more luminous main-sequence primary, and radial velocity variations are small and, therefore, difficult to detect. Since WDs are typically $\sim 10^{3}-10^{4}$ times less luminous than mainsequence stars, the brightness contrast compared to a potential faint companion is significantly reduced. Also, the markedly different spectral energy distributions of the WDs and their low-mass companions makes the detection and separation of the two components relatively straightforward even with simple broadband multicolor photometry. Observational searches for cool companions to WDs that take advantage of these factors have been carried

\footnotetext{
${ }^{1}$ Department of Physics, Harvey Mudd College, Claremont, CA 91711-5990, USA.

${ }^{2}$ Department of Physics and Astronomy, University of Southern California, Los Angeles, CA 90089, USA.

3 Department of Astronomy, New Mexico State University, Las Cruces, NM 88003-8001, USA.

4 Department of Physics, Marquette University, Milwaukee, WI 53201, USA.

5 Department of Physics, DePaul University, Chicago, IL 60614, USA.

6 School of Physics and Astronomy, University of Southampton, Southampton SO17 1BJ, UK.
}

out during the past several decades (e.g., Probst 1983; Zuckerman $\&$ Becklin 1987; Green et al. 2000).

In Wachter et al. (2003, hereafter Paper I) we presented the Two Micron All Sky Survey (2MASS; Skrutskie et al. 2006) $J H K_{s}$ photometry for all WDs from the McCook \& Sion (1999, hereafter MS99) catalog that were contained in the 2MASS Second Incremental Data Release (2IDR). We demonstrated that color selection from the near-IR color-color diagram is an effective and efficient method for identifying candidate WD + low-mass star binaries, via their near-IR excess compared to single WDs. In Paper I we recovered all 48 of the known, unresolved WD + low-mass star binaries in MS99 that were detected in the 2IDR sky coverage, and identified another 47 new candidate binaries. Our followup Hubble Space Telescope (HST) ACS snapshot survey of candidates selected largely from Paper I has borne out the efficacy of this method, through the positive identification of a large fraction of resolved binaries with angular separations as small as $\sim 0.05^{\prime \prime}$ (see the first results from this survey in Farihi et al. [2006, hereafter Paper III] and the discussion below).

In this work we present the culmination of our correlation of the MS99 catalog with the 2MASS All Sky Data Release (ASDR). The results presented here are drawn from the final calibrated photometry for the objects from Paper I, as well as the remaining targets from MS99 that were not contained in the 2IDR sky coverage. Results for the subset of magnetic WDs were presented in Wellhouse et al. (2005, hereafter Paper II), in which we did not find any strong binary candidates that could have been representative of the "missing" progenitor population of the magnetic cataclysmic variables (see discussion in Paper II and references therein). In Paper I and, especially, Paper II, we used 2MASS photometry with relatively large uncertainties, which could have led to the inclusion of several single WDs in our lists of binary candidates. In this paper we restrict our candidate sample to only those objects having 2MASS photometric uncertainties smaller than $0.1 \mathrm{mag}$ in all three bands. 


\section{THE DATA}

\subsection{Target Selection and Identification}

The source of our target sample is the MS99 catalog of optically spectroscopically identified WDs, which contains 2249 entries. Recently, a large number of new WDs have been discovered (e.g., from the Sloan Digital Sky Survey [SDSS]; Eisenstein et al. 2006). However, we restricted our potential targets to just the sample in MS99 since, as described in Paper II, the new WDs discovered by the SDSS are almost all too faint to have been detected by 2MASS. Our archival data were obtained from the 2MASS ASDR (Skrutskie et al. 2006), which includes $J H K_{s}$ images and photometry covering $99.998 \%$ of the sky. The photometric signal-to-noise ratio $(\mathrm{S} / \mathrm{N})$ is $\geq 10$ for the objects in the $2 \mathrm{MASS}$ Point Source Catalog (PSC) with $J \leq 15.8, H \leq 15.1$, and $K_{s} \leq$ 14.3. The overall detection limits of the survey are about $1 \mathrm{mag}$ fainter in each band. The PSC contains astrometry and photometry for almost 471 million objects.

Because many WDs have large (and often unknown) proper motions, their astrometry sometimes becomes unreliable only a few years after their discovery. Consequently, to identify each WD in 2MASS we first searched the literature for each object. In many cases we were able to locate published finding charts from the original, or a subsequent, identification of the $\mathrm{WD}$; for example, from the LHS atlas (Luyten \& Albers 1979), the Giclas proper-motion survey and lists of suspected WDs (e.g., Giclas 1958 through Giclas et al. 1980), and the Montreal-CambridgeTololo survey (Lamontagne et al. 2000), to name only a few sources. These were compared with all available Digitized Sky Survey (POSS-I and POSS-II) and 2MASS images in order to identify the WD (after allowing for possible proper-motion offsets, as described below).

In some cases, finding charts were not available in the literature. We then attempted to locate the WD using the most recent and accurate reported coordinates, proper motion, and/or blue optical color (e.g., by combining the POSS-II blue, red, and IR images into a three-color composite). ${ }^{7}$ For example, when data from both surveys are available for a given WD, POSS-I and POSS-II provide images spanning decades, which makes proper-motion-based identification possible for high proper motion WDs. When POSS-I images were not available, POSS-II and 2MASS images were compared for small changes in apparent position. Some Luyten Half-Second Survey objects, reported in Bakos et al. (2002), fell into this category, and a combination of proper motion and precise coordinates was used for identification. Finally, we located the entry in the 2MASS PSC corresponding to the IR counterpart in the 2MASS images. Although this process is time consuming, we estimate that simply using a coordinate match in the 2MASS PSC compared to MS99 would have resulted in up to $30 \%-40 \%$ of the WDs being misidentified or erroneously reported as undetected.

The MS99 catalog contains 2249 objects. A number of the WDs have been subsequently reclassified as non-WDs (e.g., quasars) or nonexistent (e.g., some WDs are listed twice in MS99 with different names, such as WD 2321 - 549= WD J2324-546),

\footnotetext{
${ }^{7}$ We also checked all of our targets against the finding charts in the University of Arizona White Dwarf Database, at http://procyon.lpl.arizona.edu/WD/. However, this resource is still a work in progress and somewhat incomplete, and we found a number of instances in which the wrong star was identified as the WD (for example, often in the case of a wide common proper motion pair, the non-WD component was identified as the WD). Thus, whenever possible we preferred to use this resource only to confirm our independent WD identifications rather than using it as a primary source.
}

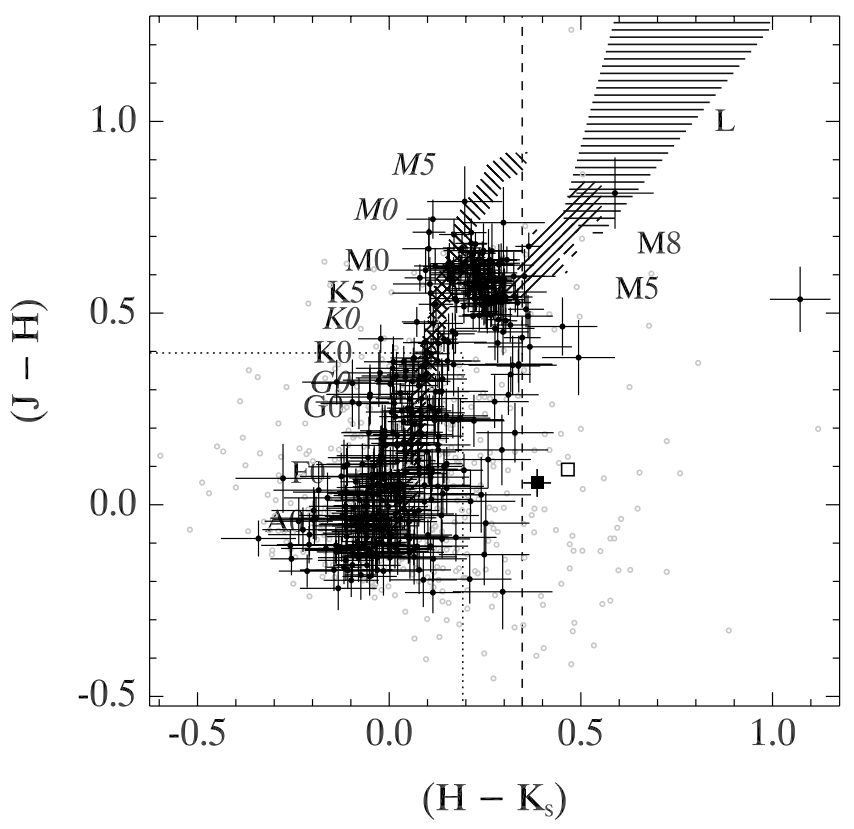

FIG. 1.-Near-IR color-color diagram for the WDs from MS99 that are detected in the 2MASS All Sky Data Release. Filled black circles with $1 \sigma$ error bars represent the good detections. Open gray circles represent the moderate detections. (Poor detections are not shown, except as noted below.) The squares represent the two known WDs with circumstellar dust disks: WD 2326+049 (G29-38; filled square, good detection) and WD 1729+371 (GD 362; open square, poor detection). The cross-hatched regions show the loci of empirical mean 2MASS colors (Bessell \& Brett 1988; Gizis et al. 2000; Kirkpatrick et al. 2000; Hawley et al. 2002; transformed from other photometric systems using the relations in Carpenter [2001] when necessary) of the main sequence (top right to bottom left diagonal hatching), giant branch (top left to bottom right diagonal hatching) and L dwarfs (horizontal hatching). Spectral types are labeled at the correct $(J-H)$ value, but offset in $\left(H-K_{s}\right)$; spectral types of the main sequence and $\mathrm{L}$ dwarfs are labeled with roman font, while those of giants are labeled with italic font. The dotted lines mark the boundary between "normal" WDs and red-excess WDs. The vertical dashed line marks the $\left(H-K_{s}\right)$ color of a dM5 star for comparison. [See the electronic edition of the Journal for a color version of this figure.]

leaving 2202 viable targets. Of these, we were unable to confidently recover the optical counterpart of the WD in 52 cases. For another 19 targets, even when we were able to recover the optical counterpart the near-IR field proved to be too crowded to confidently identify the IR counterpart. This leaves 2131 targets that we were able to confidently identify in optical and/or 2MASS images. Of these, 657 were not detected by 2MASS (i.e., not listed in the 2MASS All Sky PSC), leaving us with a final sample of 1474 WDs detected in the 2MASS ASDR.

\subsection{Near-IR Photometry and Color-Color Diagram}

As in Paper I, we graded the 2MASS photometry as "good" (all $1 \sigma$ photometric uncertainties $\leq 0.1 \mathrm{mag}$ ), "moderate" (photometric uncertainty $>0.1 \mathrm{mag}$ in one or more of the three bands), and "poor" (no formal photometric uncertainty in one or more bands, signifying a low $\mathrm{S} / \mathrm{N}$ value more properly treated as an upper limit). There are 416 good detections, 475 moderate detections, and 583 poor detections. Figure 1 shows the near-IR colorcolor diagram constructed from our data, including the $1 \sigma$ error bars of the good data. The moderate data are also shown (open gray circles), but the poor data are not plotted. Figure 2 is the same as Figure 1, except that, for clarity, we have not plotted the error bars on the good data. We restrict the remainder of our analysis and discussion to the good data.

The color-color diagrams are similar in appearance to those presented in Papers I and II. The primary difference compared 


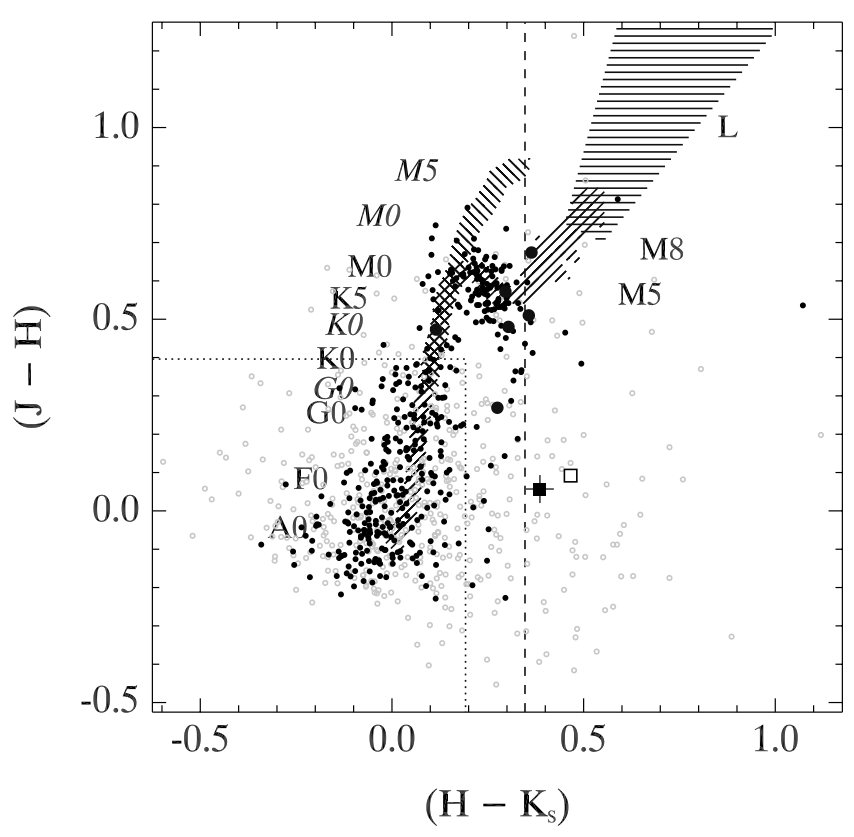

FIG. 2-Same as Fig. 1, but for clarity the error bars have not been plotted. The six objects discussed in $\S 3.2 .2$ are plotted with large symbols. [See the electronic edition of the Journal for a color version of this figure.]

to the color-color diagram in Paper I is the presence of a more populated and well-delineated "bridge" that extends almost vertically in the color-color diagram connecting the end of the WDs with colors similar to late main-sequence stars to the bluer (and presumably single) WDs. This feature can be understood in the context of the color-color diagram for simulated WD + low-mass star binaries shown in Figure 2 of Paper I, if one imagines how that figure would appear if the simulated binaries with $\left(H-K_{S}\right)>$ +0.4 were removed. Thus, the bridge noted here is possibly identified as the tail end of the color distribution of candidate binaries containing WDs and the very lowest mass companions. The tentative candidates from Paper I were drawn from this region of the color-color diagram, and (as discussed below) probably were contaminated by single WDs with red colors and/or larger photometric uncertainties.

\subsection{Binary Candidates}

The color selection boundaries used here are shown as dotted lines in the figures. They correspond to $(J-H) \geq+0.396$ (equivalent to a dK0 star) and $\left(H-K_{s}\right) \geq+0.192$. All WDs outside (i.e., redward) of these boundaries were considered as candidate binaries. The reason for choosing the $(J-H)$ boundary is discussed in Paper I; however, the choice of the $\left(H-K_{s}\right)$ boundary is somewhat more arbitrary, and was guided primarily by our estimate of the middle of the "gray zone" between the single WDs and the end of the simulated binary color distribution shown in Paper I (coincidentally, this value corresponds to the $H-K_{s}$ color of a dM0 star). Table 1 lists the 2MASS All Sky PSC photometry for all of the 153 WDs with near-IR excess selected in this fashion.

After compiling our list of WDs with near-IR excess, we reexamined the available literature for each of them in order to determine which ones are known binaries. We then sorted each WD into one of three categories: confirmed, candidate, and tentative. Confirmed binaries are those targets whose binary nature has been conclusively established in the literature, typically via spectroscopic detection of an unresolved low-mass star, resolved imaging of the binary components, and/or detailed photometric modeling showing the presence of a low-mass star spectral energy distribution after the WD is removed. Candidate binaries are those targets with near-IR excess reported, but not yet thoroughly investigated, in this work, Paper I, and/or other literature sources. Tentative candidates are those targets for which there is substantial reason to doubt that the observed near-IR colors actually indicate the presence of a cool, low-mass binary companion; for example, targets that fall into this category include DC (carbon) type WDs, possible WD + WD binaries, and WDs with less certain optical identifications. In addition, any target selected as a candidate solely because it satisfies the $\left(H-K_{S}\right)$ criterion (but not the $J-H$ criterion) was categorized as tentative if its $\left(H-K_{s}\right)$ color was within $1 \sigma$ of the $\left(H-K_{s}\right)$ color selection limit. Not surprisingly, slightly more than half (12 out of 21$)$ of the tentative candidates are drawn from the subset of WDs selected only because they satisfy the $\left(H-K_{s}\right)$ criterion.

Table 2 lists the binary status of each of the WDs from Table 1, along with some supplemental information. The columns of Table 2 show the following: Column (1) shows the WD name, as listed in Table 1. Column (2) shows the binary status, as described above. Column (3) shows the literature sources used to establish the binary status ("this work" is not listed unless it is the only source or contains crucial evidence supporting the binary status; see col. [8]). Columns (4) and (5) show estimates of the spectral types and angular separation of the binary components, if applicable (the 2MASS colors reported here are used to estimate a spectral type when possible; however, this estimate does not account for the presence of the WD, so the true spectral type is likely to be later than the nominal estimate). Column (6) shows the type of data used to establish the information in columns (4) and (5) (I, imaging; P, photometry; S, spectroscopy). Column (7) shows literature source(s) for the information in columns (4)-(6). Column (8) shows the key for the presence of additional comments about individual WDs, listed in the Appendix. Multiple rows of data in columns (4)-(7) are sometimes available for a given WD.

\subsubsection{Anomalous Objects}

Two objects stand out in the color-color diagram and deserve further explanation here (also see the corresponding notes in the Appendix). WD 1201+437 is the object located at extremely red $\left(H-K_{S}\right)$ color and is possibly a quasar. WD $1517+502$ is the only object located in the L spectral type region of the color-color diagram, but it is a known binary in which the WD's companion is a dwarf carbon star, not an L dwarf.

WD 2326+049 (= G29-38) formally qualifies for inclusion in our list of color-selected binary candidates, but has been excluded from the tables in this work because it is known to be surrounded by a dust disk (e.g., Graham et al. 1990; Tokunaga et al. 1990; Jura 2003; Reach et al. 2005), and the presence of binary companions has been excluded down to angular separations of $a \geq 0.9^{\prime \prime}$ and masses of $M_{2} \geq 6 M_{\mathrm{J}}$ (Debes et al. 2005a). For comparison with our known and candidate binaries, we have plotted the 2MASS colors of WD 2326+049 and the other known WD with a dust disk (WD 1729+371 = GD362; Becklin et al. 2005) with special symbols in the figures. The near-IR colors of these two WDs with dust disks are similar to each other. They satisfy only the $\left(H-K_{S}\right)$ color selection criterion, but fall outside the expected locus for $\mathrm{WD}+$ very low mass companion binaries shown in the simulation from Paper I. There are several WDs whose $1 \sigma$ uncertainty ranges overlap with the WD + dust disk colors, but none whose nominal colors match. 
TABLE 1

2MASS Photometry for White Dwarfs with Near-IR Excess

\begin{tabular}{|c|c|c|c|c|}
\hline WD & 2MASS & $J$ & $H$ & $K_{s}$ \\
\hline $0014+097 \ldots \ldots$. & $00165618+1003591$ & $12.414(25)$ & $11.880(29)$ & $11.602(22)$ \\
\hline $0017+061 .$. & $00194100+0624075$ & $13.738(33)$ & $13.189(34)$ & $12.983(32)$ \\
\hline 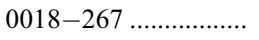 & $00213073-2626114$ & $12.504(26)$ & $12.106(25)$ & $12.006(23)$ \\
\hline $0023+388$. & $00263309+3909044$ & $13.810(26)$ & $13.268(30)$ & $12.939(33)$ \\
\hline $0027-549 \ldots \ldots \ldots \ldots \ldots \ldots$ & $00294996-5441354$ & $9.758(24)$ & $9.129(23)$ & $8.883(23)$ \\
\hline $0034-211 \ldots$ & $00372502-2053422$ & $11.454(23)$ & $10.884(21)$ & $10.648(26)$ \\
\hline $0041+092$. & $00440131+0932578$ & $8.450(34)$ & $7.927(34)$ & $7.805(29)$ \\
\hline 0104-331. & $01064686-3253124$ & $14.740(38)$ & $14.162(44)$ & $13.913(57)$ \\
\hline $0116-231 \ldots \ldots \ldots \ldots \ldots \ldots$ & $01183718-2254578$ & $14.640(42)$ & $14.100(37)$ & $13.828(55)$ \\
\hline $0130-196 \ldots$ & $01323935-1921394$ & $14.749(37)$ & $14.253(32)$ & $13.997(57)$ \\
\hline $0131-163 .$. & $01342407-1607083$ & $12.966(27)$ & $12.468(28)$ & $12.215(30)$ \\
\hline $0145-705$. & $01461179-7020197$ & $14.962(41)$ & $14.538(63)$ & $14.383(77)$ \\
\hline $0145-221 \ldots \ldots \ldots \ldots \ldots \ldots$ & $01472183-2156512$ & $14.923(32)$ & $14.450(45)$ & $14.335(64)$ \\
\hline $0148-255 \mathrm{~J} .$. & $01480822-2532452$ & $12.412(26)$ & $11.830(21)$ & $11.594(23)$ \\
\hline $0205+133 \ldots \ldots$ & $02080350+1336256$ & $12.799(22)$ & $12.198(24)$ & $11.961(20)$ \\
\hline $0208-153$. & $02104280-1506356$ & $12.589(24)$ & $12.057(25)$ & $11.772(25)$ \\
\hline $0232+035 \ldots \ldots$ & $02350758+0343567$ & $11.265(24)$ & $10.733(21)$ & $10.557(19)$ \\
\hline $0237+115 \ldots \ldots$ & $02400663+1148280$ & $13.788(30)$ & $13.106(27)$ & $12.892(32)$ \\
\hline $0248+601 \ldots \ldots \ldots \ldots \ldots \ldots$ & $02520803+6019428$ & $13.529(21)$ & $12.894(28)$ & $12.673(29)$ \\
\hline 0254-053J ................ & $02543883-0519509$ & $5.709(19)$ & $5.263(47)$ & $5.090(18)$ \\
\hline $0255+009.2$. & $02581788+0109458$ & $15.560(63)$ & $14.824(69)$ & $14.526(83)$ \\
\hline $0258+184 \ldots \ldots \ldots \ldots \ldots \ldots$ & $03011287+1840539$ & $14.991(40)$ & $14.803(67)$ & $14.475(76)$ \\
\hline $0303-007$.................. & $03060719-0031144$ & $13.164(24)$ & $12.627(27)$ & $12.405(26)$ \\
\hline $0302+621 \ldots \ldots .$. & $03061669+6222226$ & $15.015(44)$ & $14.989(89)$ & $14.749(95)$ \\
\hline $0308+096 \ldots \ldots$. & $03105499+0949256$ & $13.723(29)$ & $13.183(31)$ & $12.934(35)$ \\
\hline 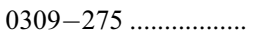 & $03113318-2719260$ & $13.514(26)$ & $12.885(34)$ & $12.731(30)$ \\
\hline $0312+019 \ldots$ & $03145212+0206072$ & $15.603(51)$ & $14.998(55)$ & $14.776(98)$ \\
\hline $0347-137 \ldots \ldots$ & $03501451-1335138$ & $12.080(29)$ & $11.540(29)$ & $11.296(23)$ \\
\hline $0357+286 \mathrm{~J} \ldots \ldots$ & $03570582+2837516$ & $9.843(23)$ & $9.275(24)$ & $9.057(17)$ \\
\hline $0354+463 \ldots \ldots$ & $03581711+4628397$ & $13.594(27)$ & $13.084(38)$ & $12.727(27)$ \\
\hline $0357-233$ & $03590491-2312243$ & $14.961(44)$ & $14.594(60)$ & $14.256(65)$ \\
\hline $0357+081 \ldots \ldots$ & $04002668+0814069$ & $14.562(38)$ & $14.343(56)$ & $14.122(57)$ \\
\hline $0413-077 \ldots$. & $04152173-0739173$ & $6.747(20)$ & $6.278(40)$ & $5.962(26)$ \\
\hline $0416+272 \ldots \ldots$ & $04185663+2717484$ & $15.134(38)$ & $15.361(90)$ & $15.065(94)$ \\
\hline $0419-487 \ldots \ldots$ & $04210556-4839070$ & $10.720(24)$ & $10.148(23)$ & $9.852(25)$ \\
\hline $0429+176 \ldots \ldots$ & $04322373+1745026$ & $10.753(21)$ & 10.161(19) & $9.913(17)$ \\
\hline $0430+136 \ldots$ & $04331053+1345134$ & $13.533(21)$ & $12.877(23)$ & $12.634(26)$ \\
\hline $0458-662$. & $04585395-6628134$ & $13.437(26)$ & $12.727(27)$ & $12.513(29)$ \\
\hline $0627+299 \ldots \ldots$ & $06303679+2956180$ & $15.128(38)$ & $15.176(71)$ & $14.924(89)$ \\
\hline $0628-020 \ldots \ldots \ldots \ldots \ldots$ & $06303881-0205537$ & $10.729(27)$ & $10.144(26)$ & $9.857(24)$ \\
\hline $0710+741 \ldots \ldots$. & $07170975+7400406$ & $14.692(33)$ & $14.423(61)$ & $14.148(65)$ \\
\hline $0718-316$. & $07204790-3147027$ & $13.253(25)$ & $12.749(26)$ & $12.502(27)$ \\
\hline $0752-146 \ldots \ldots \ldots \ldots \ldots$ & $07550896-1445506$ & $12.621(24)$ & $12.141(23)$ & $11.837(19)$ \\
\hline $0800-533 \ldots \ldots \ldots \ldots \ldots . . . . . . .$. & $08020022-5327499$ & $13.703(33)$ & $13.186(27)$ & $12.991(27)$ \\
\hline $0802+387 \ldots \ldots$. & $08055764+3833444$ & $15.336(47)$ & 15.193(79) & $14.899(91)$ \\
\hline $0805+654 \ldots \ldots$ & $08094545+6518172$ & $14.002(24)$ & $13.449(27)$ & $13.171(31)$ \\
\hline $0812+478 \ldots \ldots \ldots \ldots \ldots \ldots$ & $08154894+4740391$ & $14.587(32)$ & $14.165(41)$ & $13.882(47)$ \\
\hline $0824+288 \ldots \ldots$ & $08270508+2844024$ & $12.423(28)$ & $11.802(28)$ & $11.650(30)$ \\
\hline $0852+630 \ldots \ldots$ & $08562826+6250226$ & $12.827(24)$ & $12.122(33)$ & $11.953(21)$ \\
\hline $0858-220$. & $09005722-2213505$ & $11.348(27)$ & $10.771(24)$ & $10.572(21)$ \\
\hline $0908+226 \ldots \ldots$. & $09114308+2227488$ & $15.160(40)$ & $14.492(35)$ & $14.389(59)$ \\
\hline $0915+201 \ldots \ldots$. & $09183291+1953070$ & $15.721(58)$ & $15.166(78)$ & $14.867(80)$ \\
\hline $0928+399 \ldots$ & $09315566+3946076$ & $15.049(41)$ & $14.453(58)$ & $14.100(56)$ \\
\hline $0933+025 \ldots \ldots \ldots \ldots \ldots$ & $09354067+0222005$ & $13.270(24)$ & $12.726(23)$ & $12.477(24)$ \\
\hline 0937-095 „................ & $09394969-0945562$ & $14.715(33)$ & $14.135(38)$ & $13.898(48)$ \\
\hline $0949+451 \ldots \ldots \ldots \ldots \ldots \ldots$ & $09522209+4454288$ & $11.924(22)$ & $11.335(16)$ & $11.035(13)$ \\
\hline $0950+185 \ldots \ldots \ldots \ldots \ldots \ldots . .$. & $09524582+1821026$ & $12.691(22)$ & $12.011(22)$ & $11.786(20)$ \\
\hline $0956+045 \ldots \ldots$ & $09583717+0421292$ & $14.659(40)$ & $14.131(46)$ & $13.837(59)$ \\
\hline $1001+203 \ldots \ldots \ldots \ldots \ldots \ldots$ & $10040431+2009226$ & $12.640(21)$ & $12.028(21)$ & $11.766(20)$ \\
\hline 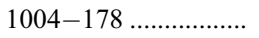 & $10070776-1805246$ & $12.555(22)$ & $11.983(27)$ & $11.745(23)$ \\
\hline $1013-050 \ldots \ldots \ldots \ldots \ldots . .$. & $10162867-0520320$ & $10.607(27)$ & $9.990(25)$ & $9.770(23)$ \\
\hline 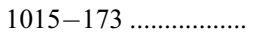 & $10172883-1737059$ & $15.238(50)$ & $14.874(58)$ & $14.552(99)$ \\
\hline $1026+002 \ldots \ldots \ldots \ldots \ldots \ldots$ & $10283487-0000295$ & $11.751(24)$ & $11.219(27)$ & $10.943(21)$ \\
\hline $1027-039$.................. & $10295925-0413014$ & $15.207(50)$ & $14.611(38)$ & $14.286(71)$ \\
\hline $1033+464 \ldots$. & $10362522+4608312$ & $12.564(22)$ & $12.032(24)$ & $11.752(18)$ \\
\hline
\end{tabular}


TABLE 1-Continued

\begin{tabular}{|c|c|c|c|c|}
\hline WD & 2MASS & $J$ & $H$ & $K_{s}$ \\
\hline $1036-204$. & $10385559-2040572$ & $14.633(33)$ & $14.346(41)$ & $14.035(67)$ \\
\hline $1037+512 \ldots \ldots \ldots \ldots \ldots$ & $10401680+5056468$ & $13.796(24)$ & $13.261(26)$ & $12.972(26)$ \\
\hline $1042-690$..................... & $10441023-6918180$ & $11.423(26)$ & $10.896(27)$ & $10.561(21)$ \\
\hline $1049+103 \ldots \ldots \ldots \ldots \ldots$ & $10522772+1003380$ & $13.266(29)$ & $12.830(35)$ & $12.483(33)$ \\
\hline $1055-072$. & $10573517-0731233$ & $13.770(29)$ & $13.680(32)$ & $13.485(38)$ \\
\hline $1101+364 \ldots$ & $11043257+3610490$ & $14.832(38)$ & $14.962(70)$ & $14.714(95)$ \\
\hline $1104+044 \ldots .$. & $11070472+0409077$ & $14.127(33)$ & $13.674(35)$ & $13.509(53)$ \\
\hline $1106+316 \ldots \ldots \ldots \ldots \ldots$ & $11084306+3123559$ & $15.136(41)$ & $14.524(44)$ & $14.429(86)$ \\
\hline 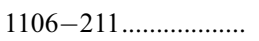 & $11091095-2123320$ & $14.676(33)$ & $13.931(39)$ & $13.817(57)$ \\
\hline $1123+189 \ldots \ldots \ldots \ldots \ldots \ldots$ & $11261906+1839178$ & $12.754(23)$ & $12.217(19)$ & $11.990(20)$ \\
\hline $1126+185 \ldots \ldots \ldots \ldots \ldots$ & $11291804+1816457$ & $12.643(23)$ & $12.166(31)$ & $12.094(23)$ \\
\hline 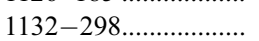 & $11352186-3010156$ & $13.799(32)$ & $13.164(29)$ & $12.964(35)$ \\
\hline $1133+358 \ldots \ldots \ldots \ldots \ldots \ldots$ & $11354300+3534235$ & $11.625(19)$ & $11.079(21)$ & $10.802(19)$ \\
\hline $1136+667 \ldots \ldots \ldots \ldots \ldots \ldots$ & $11390593+6630184$ & $12.314(25)$ & $11.695(26)$ & $11.543(23)$ \\
\hline $1141+504 \ldots \ldots \ldots \ldots \ldots \ldots$ & $11434997+5010203$ & $14.779(40)$ & $14.215(43)$ & $13.989(48)$ \\
\hline 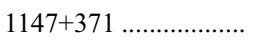 & $11503125+3654159$ & $14.892(39)$ & $14.271(48)$ & $14.114(48)$ \\
\hline 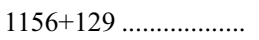 & $11591564+1239299$ & $14.721(32)$ & $14.099(30)$ & $13.895(42)$ \\
\hline $1201+437 \ldots$ & $12042403+4330570$ & $15.393(51)$ & $14.857(68)$ & $13.785(41)$ \\
\hline $1210+464 \ldots$. & $12125961+4609467$ & $12.035(23)$ & $11.396(21)$ & $11.161(20)$ \\
\hline $1213+528 \ldots \ldots \ldots \ldots \ldots \ldots$ & $12154411+5231013$ & $9.979(22)$ & $9.340(27)$ & $9.033(21)$ \\
\hline $1214+032 \ldots \ldots \ldots \ldots \ldots \ldots$ & $12165190+0258046$ & $9.234(18)$ & $8.671(22)$ & $8.422(18)$ \\
\hline $1218+497$..................... & $12210535+4927207$ & $14.588(38)$ & $14.002(36)$ & $13.837(60)$ \\
\hline $1224+309 \ldots \ldots \ldots \ldots \ldots \ldots$ & $12263089+3038527$ & $15.129(48)$ & $14.669(68)$ & $14.393(77)$ \\
\hline $1246+299 \ldots \ldots \ldots \ldots \ldots \ldots$ & $12484722+2942507$ & $15.055(43)$ & $14.479(55)$ & $14.373(79)$ \\
\hline $1247-176$ & $12502208-1754465$ & $13.502(24)$ & $12.863(23)$ & $12.601(31)$ \\
\hline $1255+258 \mathrm{~J} \ldots \ldots \ldots \ldots \ldots \ldots$ & $12553374+2553308$ & $7.370(27)$ & $6.947(51)$ & $6.855(24)$ \\
\hline $1254-133$.................... & $12563956-1334421$ & $14.499(33)$ & $13.973(51)$ & $13.706(51)$ \\
\hline $1305+018 \ldots \ldots \ldots \ldots \ldots \ldots$ & $13075472+0132106$ & $12.982(26)$ & $12.400(23)$ & $12.144(21)$ \\
\hline $1307-141$...................... & $13102255-1427099$ & $13.849(23)$ & $13.226(35)$ & $13.013(36)$ \\
\hline 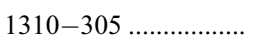 & $13134158-3051336$ & $14.931(35)$ & $15.125(53)$ & $14.915(95)$ \\
\hline 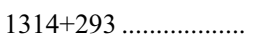 & $13162169+2905548$ & $10.373(19)$ & $9.807(29)$ & $9.565(25)$ \\
\hline 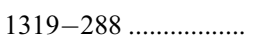 & $13224044-2905347$ & $12.754(24)$ & $12.271(23)$ & $11.986(23)$ \\
\hline 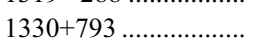 & $13303294+7905126$ & $12.506(21)$ & $11.880(23)$ & $11.657(19)$ \\
\hline $1333+487 \ldots \ldots \ldots \ldots \ldots \ldots$ & $13360209+4828472$ & $11.794(23)$ & $11.200(17)$ & $10.927(23)$ \\
\hline $1340+604 \mathrm{~J} \ldots \ldots \ldots \ldots \ldots \ldots$ & $13410002+6026104$ & $15.555(58)$ & $15.143(74)$ & $14.776(82)$ \\
\hline $1401+005 \ldots \ldots \ldots \ldots \ldots \ldots$ & $14034531+0021359$ & $13.443(23)$ & $12.851(26)$ & $12.771(30)$ \\
\hline $1412-049$..................... & $14150220-0511040$ & $13.803(30)$ & $13.092(27)$ & $12.988(34)$ \\
\hline $1412-109$................... & $14150761-1109213$ & $15.379(64)$ & $14.588(66)$ & $14.391(73)$ \\
\hline $1415+132 \ldots \ldots \ldots \ldots \ldots$ & $14174025+1301487$ & $14.263(36)$ & $13.725(46)$ & $13.553(46)$ \\
\hline $1424+503 \ldots \ldots \ldots \ldots \ldots \ldots$ & $14264410+5006274$ & $10.626(20)$ & $10.008(15)$ & $9.822(22)$ \\
\hline $1433+538 \ldots \ldots \ldots \ldots \ldots \ldots$ & $14344329+5335212$ & $14.667(35)$ & $14.216(50)$ & $13.919(45)$ \\
\hline 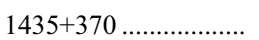 & $14373667+3651378$ & $13.457(24)$ & $12.965(25)$ & $12.746(28)$ \\
\hline 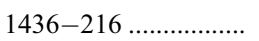 & $14391264-2150138$ & $13.312(27)$ & $12.770(22)$ & $12.519(29)$ \\
\hline $1443+336 \ldots \ldots \ldots \ldots \ldots \ldots$ & $14460066+3328502$ & $14.284(30)$ & $13.725(30)$ & $13.516(40)$ \\
\hline 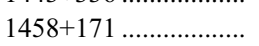 & $15001934+1659146$ & $14.701(31)$ & $14.209(45)$ & $13.847(47)$ \\
\hline $1501+300 \ldots \ldots \ldots \ldots \ldots \ldots$ & $15032075+2952584$ & $14.178(27)$ & $13.745(26)$ & $13.767(53)$ \\
\hline $1502+349 \ldots \ldots \ldots \ldots \ldots \ldots$ & $15043185+3446584$ & $15.231(45)$ & $14.766(61)$ & $14.314(67)$ \\
\hline $1504+546 \ldots \ldots \ldots \ldots \ldots \ldots$ & $15060542+5428186$ & $13.847(25)$ & $13.260(26)$ & $13.001(27)$ \\
\hline $1517+502 \ldots \ldots \ldots \ldots \ldots \ldots$ & $15190599+5007027$ & $15.559(60)$ & $14.746(71)$ & $14.157(72)$ \\
\hline 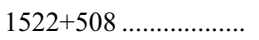 & $15242519+5040098$ & $14.757(35)$ & $14.141(45)$ & $13.959(58)$ \\
\hline $1541-381 \ldots \ldots \ldots \ldots \ldots$ & $15451092-3818515$ & $11.953(25)$ & $11.432(28)$ & $11.190(25)$ \\
\hline $1558+616 \ldots \ldots \ldots \ldots \ldots \ldots$ & $15585553+6132037$ & $14.204(32)$ & $13.600(42)$ & $13.368(42)$ \\
\hline 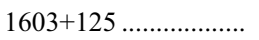 & $16053211+1225429$ & $13.552(23)$ & $13.120(23)$ & $12.977(26)$ \\
\hline 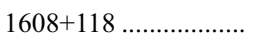 & $16105319+1143538$ & $12.059(22)$ & $11.482(23)$ & $11.260(19)$ \\
\hline $1610+383 \ldots \ldots \ldots \ldots \ldots \ldots$ & $16122147+3812299$ & $14.437(34)$ & $13.807(36)$ & $13.521(42)$ \\
\hline 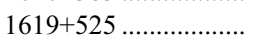 & $16202428+5223215$ & $14.168(32)$ & $13.545(35)$ & $13.425(42)$ \\
\hline $1619+414$ & $16211268+4118093$ & $13.937(21)$ & $13.311(29)$ & $13.025(27)$ \\
\hline $1622+323 \ldots \ldots \ldots \ldots \ldots \ldots$ & $16244899+3217021$ & $14.633(29)$ & $13.963(31)$ & $13.773(39)$ \\
\hline $1631+781 \ldots \ldots \ldots \ldots \ldots \ldots$ & $16291031+7804399$ & $10.975(21)$ & $10.398(21)$ & $10.164(14)$ \\
\hline $1632-227.1 \ldots \ldots \ldots \ldots . . .$. & $16352428-2250279$ & $15.279(56)$ & $14.654(75)$ & $14.395(92)$ \\
\hline $1634-573 \ldots \ldots \ldots \ldots \ldots \ldots$ & $16383108-5728112$ & $7.120(23)$ & $6.699(44)$ & $6.571(29)$ \\
\hline 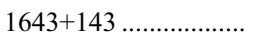 & $16453913+1417462$ & $12.732(24)$ & $12.125(31)$ & $11.957(24)$ \\
\hline $1646+062 \ldots \ldots \ldots \ldots \ldots$ & $16490776+0608453$ & $14.035(27)$ & $13.424(30)$ & $13.237(35)$ \\
\hline 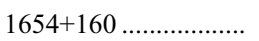 & $16565765+1556254$ & $13.059(29)$ & $12.421(33)$ & $12.129(24)$ \\
\hline 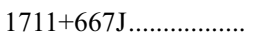 & $17112736+6645319$ & $15.120(43)$ & $14.457(57)$ & $14.211(87)$ \\
\hline $1717-345 \ldots \ldots \ldots \ldots \ldots$ & $17211032-3433286$ & $12.870(39)$ & $12.208(60)$ & $11.940(54)$ \\
\hline
\end{tabular}


TABLE 1-Continued

\begin{tabular}{|c|c|c|c|c|}
\hline WD & 2MASS & $J$ & $H$ & $K_{s}$ \\
\hline $1820+580 \mathrm{~J} \ldots \ldots$. & $18202977+5804410$ & $14.058(32)$ & $13.718(35)$ & $13.401(39)$ \\
\hline $1833+644 \ldots \ldots \ldots \ldots \ldots$ & $18332921+6431520$ & $14.098(30)$ & $13.516(30)$ & $13.241(38)$ \\
\hline $1845+019 \ldots \ldots \ldots \ldots \ldots$ & $18473908+0157356$ & $12.398(53)$ & $12.014(82)$ & $11.520(47)$ \\
\hline $1844-654 \ldots \ldots$ & $18490202-6525144$ & $12.703(24)$ & $12.045(26)$ & $11.833(21)$ \\
\hline $1950+279$. & $19522838+2807527$ & $15.187(48)$ & $14.635(60)$ & $14.527(80)$ \\
\hline $2009+622$. & $20104287+6225321$ & $14.277(31)$ & $13.915(31)$ & $13.578(48)$ \\
\hline $2013+400 \mathrm{~J} \ldots \ldots \ldots \ldots \ldots$ & $20130936+4002242$ & $13.044(24)$ & $12.520(24)$ & $12.260(32)$ \\
\hline $2024+200 \mathrm{~J} \ldots \ldots \ldots \ldots \ldots . . . . . . . . .$. & $20241609+2000474$ & $14.370(33)$ & $13.845(43)$ & $13.595(47)$ \\
\hline $2101-364 \ldots \ldots \ldots \ldots \ldots$ & $21044692-3615251$ & $14.515(26)$ & $13.915(32)$ & $13.756(38)$ \\
\hline $2108-431 \ldots \ldots \ldots \ldots \ldots . .$. & $21113740-4258116$ & $12.773(24)$ & $12.161(23)$ & $11.934(25)$ \\
\hline $2118-333 \ldots \ldots \ldots \ldots \ldots$ & $21214687-3310477$ & $12.595(24)$ & $11.950(25)$ & $11.734(25)$ \\
\hline $2131+066 \ldots \ldots \ldots \ldots$ & $21340822+0650573$ & $15.317(42)$ & $14.723(60)$ & $14.486(92)$ \\
\hline $2133+463 \ldots \ldots \ldots \ldots \ldots \ldots$ & $21351760+4633174$ & $11.297(21)$ & $10.756(17)$ & $10.460(19)$ \\
\hline $2151-015 \ldots \ldots \ldots \ldots$ & $21540644-0117102$ & $12.452(29)$ & $11.778(22)$ & $11.414(27)$ \\
\hline $2154+408 \ldots \ldots \ldots \ldots \ldots$ & $21561824+4102452$ & $12.877(25)$ & $12.383(33)$ & $12.148(27)$ \\
\hline $2237+819$ & $22371556+8210273$ & $12.824(26)$ & $12.282(32)$ & $11.978(24)$ \\
\hline $2256+249 \ldots \ldots \ldots \ldots \ldots$ & $22584811+2515439$ & $11.675(20)$ & $11.180(25)$ & $10.915(18)$ \\
\hline $2311-068 \ldots \ldots \ldots \ldots \ldots$ & $23142520-0632475$ & $14.951(36)$ & $14.942(71)$ & $14.730(93)$ \\
\hline $2317+268 \ldots \ldots \ldots \ldots$ & $23200401+2706237$ & $14.609(33)$ & $14.074(36)$ & $13.783(50)$ \\
\hline $2318-137 \ldots \ldots \ldots \ldots \ldots \ldots$ & $23210825-1327465$ & $12.074(22)$ & $11.444(24)$ & $11.174(21)$ \\
\hline $2326-224 \ldots \ldots \ldots \ldots \ldots$ & $23283886-2210209$ & $12.627(26)$ & $12.035(23)$ & $11.766(21)$ \\
\hline $2336-187 \ldots \ldots \ldots \ldots \ldots$ & $23385279-1826123$ & $15.057(40)$ & $14.939(63)$ & $14.681(93)$ \\
\hline
\end{tabular}

Noте.-Table 1 is also available in machine-readable form in the electronic edition of the Astronomical Journal.

\subsection{Number Statistics}

Table 3 lists the total number of WDs with near-IR excess reported in this work, as well as the break-down into each of the three binary status categories used in Table 2 (i.e., confirmed, candidate, and tentative). We also show the numbers of WDs that are newly reported in this work and the current status of the binary candidates/WDs with near-IR excess reported in Paper I. Of the 153 objects reported in this work, 54\% are new, and the remainder were first reported in Paper I. At the time of Paper I, only about one-half of our reported WDs with near-IR excess were already known to be binaries, but in the intervening years more work has been accomplished in this field, and the fraction of confirmed binaries (as reported in the literature) from our total sample in this work is close to $70 \%$. For example, 20 of the candidates from Paper I were subsequently confirmed by us to be binaries at small angular separations that are resolvable by HST ACS (Paper III). This leaves 27 firm binary candidates (19 of which are new to this work) and 21 tentative candidates (17 of which are new to this work).

Of the 95 candidates from Paper I, 27 are "missing" from our current sample of WDs with near-IR excess. Fifteen of these were removed because of better identification information ${ }^{8}$ that leaves them as either undetected by 2MASS or with their identifications uncertain. Another nine were excluded from the current candidate list because they have only moderate or poor 2MASS detections. The remaining three "missing" WDs are equally divided among being reclassified as something other than a WD, not satisfying the red selection criteria with the recalibrated All Sky photometry, and not being included in the All Sky PSC. Of the 15 tentative candidates from Paper I, all but three have been excluded from the current candidate list because they have only moderate 2 MASS detections.

\footnotetext{
${ }^{8}$ A few of these were noted independently in Tremblay \& Bergeron (2007).
}

\section{ANALYSIS AND DISCUSSION}

\subsection{Discussion of the Synthetic Photometry Method}

Tremblay \& Bergeron (2007) have recently suggested that a method involving the calculation of synthetic photometry from WD model atmospheres is more efficient than our color selection technique as a means to identify WDs with near-IR excess that are binary candidates. While the former method is undeniably effective and possibly has a lower "false positive" rate, it requires multiband optical and near-IR photometry for each target, and is also more computationally and analytically complex than simple color selection from the preexisting, homogenous 2MASS PSC. Consequently, we suggest that the latter method is, in fact, the more efficient method of identifying binary candidates, in terms of simplicity and ease of use (also see $\S 3.2 .1$ ). The synthetic photometry method is likely more effective at providing subsequent confirmation and characterization of the near-IR excess in color-selected binary candidates (e.g., see discussion of WD $0145-221$ in $\S 3.2 .2$ ).

\subsection{Comparison with the Paper I Results \\ 3.2.1. Current Status of Past Binary Candidates}

Overall, our "success" rate in finding binary candidates using 2MASS color selection that were subsequently confirmed as binaries is very high. For example, in Paper I we reported 47 new binary candidates (excluding the tentative candidates). Eighteen of these have now been excluded from our sample for reasons other than simply having moderate or poor $2 \mathrm{MASS}$ detections (see $\S 2.4$ ). Of the remaining 29, 28 have been confirmed as binaries by us in Paper III via high angular resolution imaging (20 objects) or using a method similar to that described by Tremblay \& Bergeron (2007; eight objects). So, our confirmed success rate is $80 \%$ (including the 48 already known binaries recovered as part of the original 95 candidates in Paper I), while our confirmed false positive rate (for targets that have been 
TABLE 2

Binary Parameters

\begin{tabular}{|c|c|c|c|c|c|c|c|}
\hline $\begin{array}{l}\text { WD } \\
\text { (1) }\end{array}$ & $\begin{array}{c}\text { Binary Status } \\
\text { (2) }\end{array}$ & $\begin{array}{c}\text { Binary Status References } \\
\text { (3) }\end{array}$ & $\begin{array}{l}\text { Spectral Types } \\
\text { (4) }\end{array}$ & $\begin{array}{c}a_{\text {sep }} \\
(\operatorname{arcsec}) \\
(5)\end{array}$ & $\begin{array}{l}\text { Data } \\
(6)\end{array}$ & $\begin{array}{c}\text { References } \\
\text { for Cols. (4)-(6) } \\
\text { (7) }\end{array}$ & $\begin{array}{c}\text { Comments } \\
\text { (8) }\end{array}$ \\
\hline \multirow[t]{2}{*}{ 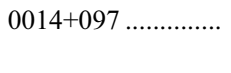 } & Confirmed & 36 & $\mathrm{WD}+\mathrm{dM}$ & $\ldots$ & $\mathrm{S}$ & 36 & $\ldots$ \\
\hline & & & $\mathrm{dM} 2-4$ & $\lesssim 4$ & IP & This work & $\ldots$ \\
\hline \multirow[t]{2}{*}{$0017+061 \ldots \ldots \ldots \ldots . .}$. & Confirmed & 7,8 & $\mathrm{DA} 2+\mathrm{dM} 4$ & 2.0 & IP & 8 & $\ldots$ \\
\hline & & & $\mathrm{dM} 0-2.5$ & $\lesssim 4$ & IP & This work & $\ldots$ \\
\hline $0018-267 \ldots \ldots \ldots \ldots$ & Tentative & This work & $\mathrm{dG} 8-\mathrm{K} 2$ & $\lesssim 4$ & IP & This work & See Appendix \\
\hline \multirow[t]{2}{*}{$0023+388 \ldots \ldots \ldots \ldots \ldots$} & Confirmed & 43, Papers I and III & WD+dM5.5 & $\lesssim 0.025$ & IP & Paper III & See Appendix \\
\hline & & & dM3-5 & $\lesssim 4$ & IP & This work & $\ldots$ \\
\hline \multirow[t]{2}{*}{$0027-549 \ldots \ldots \ldots \ldots$} & Confirmed & 47,62 & $\mathrm{DA}+\mathrm{dM} 3$ & 4 & IS & 47,62 & $\ldots$ \\
\hline & & & $\mathrm{dM} 1-2.5$ & $\lesssim 4$ & IP & This work & $\ldots$ \\
\hline \multirow[t]{3}{*}{$0034-211 \ldots \ldots \ldots \ldots \ldots$} & Confirmed & 8, 43, Papers I and III & $\mathrm{WD}+\mathrm{dM} 3.5$ & 0.328 & IP & Paper III & See Appendix \\
\hline & & & DA3+dM3 & $\leq 0.5$ & IP & 8 & $\ldots$ \\
\hline & & & $\mathrm{dM} 1-3$ & $\lesssim 4$ & IP & This work & $\ldots$ \\
\hline \multirow{3}{*}{$0041+092 \ldots \ldots \ldots \ldots . . .}$. & Confirmed & 30,43 & $\mathrm{DA}+\mathrm{dK} 2$ & $\lesssim 0.08$ & IP & 2 & See Appendix \\
\hline & & & $\mathrm{DA}+\mathrm{dK} 1-3$ & $<1.9$ & IPS & 30 & $\ldots$ \\
\hline & & & $\mathrm{dK} 3-5$ & $\lesssim 4$ & IP & This work & $\ldots$ \\
\hline $0104-331 \ldots \ldots \ldots \ldots$ & Candidate & This work & $\mathrm{dM} 0-4.5$ & $\lesssim 4$ & IP & This work & $\ldots$ \\
\hline \multirow{2}{*}{$0116-231 \ldots \ldots \ldots \ldots$} & Confirmed & Papers I and III & $\mathrm{WD}+\mathrm{dM} 4.5$ & 1.105 & IP & Paper III & See Appendix \\
\hline & & & $\mathrm{dM} 1-5$ & $\lesssim 4$ & IP & This work & $\ldots$ \\
\hline $0130-196 \ldots \ldots \ldots \ldots$ & Candidate & Paper I & $\mathrm{dM} 0-4.5$ & $\lesssim 4$ & IP & This work & See Appendix \\
\hline \multirow[t]{3}{*}{$0131-163 \ldots \ldots \ldots \ldots \ldots$} & Confirmed & 8, Papers I and III & WD+dM3.5 & 0.189 & IP & Paper III & $\ldots$ \\
\hline & & & $\mathrm{DA} 1+\mathrm{dM} 2$ & $\leq 0.5$ & IP & 8 & $\ldots$ \\
\hline & & & $\mathrm{dM} 1-3$ & $\lesssim 4$ & IP & This work & $\ldots$ \\
\hline $0145-705 \ldots \ldots \ldots \ldots$ & Candidate & This work & dG6-dK3 & $\lesssim 4$ & IP & This work & $\ldots$ \\
\hline \multirow[t]{2}{*}{$0145-221 \ldots \ldots \ldots \ldots$} & Confirmed & 8, Paper I & DA4+dL6 & $\leq 0.3$ & IP & 8 & $\ldots$ \\
\hline & & & $\mathrm{dK} 1-4$ & $\lesssim 4$ & IP & This work & $\ldots$ \\
\hline \multirow[t]{3}{*}{ J0148-255 ............ } & Confirmed & $14,43,45,79$, Papers I and III & WD+dM3.5 & 2.295 & IP & Paper III & See Appendix \\
\hline & & & $\mathrm{dM} 1-3$ & $\lesssim 4$ & IP & This work & $\ldots$ \\
\hline & & & $\mathrm{WD}+\mathrm{dM} 3-4$ & $\lesssim 10$ & IP & 14 & $\ldots$ \\
\hline \multirow[t]{2}{*}{$0205+133 \ldots \ldots \ldots \ldots$} & Confirmed & 15, Papers I and III & WD+dM1 & 1.257 & IP & Paper III & $\ldots$ \\
\hline & & & $\mathrm{dM} 0-3$ & $\lesssim 4$ & IP & This work & $\ldots$ \\
\hline \multirow[t]{2}{*}{$0208-153 \ldots \ldots \ldots \ldots$} & Confirmed & Papers I and III & $\mathrm{WD}+\mathrm{dM} 2$ & 2.647 & IP & Paper III & $\ldots$ \\
\hline & & & $\mathrm{dM} 2.5-4.5$ & $\lesssim 4$ & IP & This work & $\ldots$ \\
\hline \multirow[t]{2}{*}{$0232+035 \ldots \ldots \ldots \ldots \ldots$} & Confirmed & 43,66 & $\mathrm{DA}+\mathrm{dM}$ & Close & $\mathrm{S}$ & 18,66 & See Appendix \\
\hline & & & $\mathrm{dK} 3-\mathrm{M} 1$ & $\lesssim 4$ & IP & This work & $\ldots$ \\
\hline $0237+115 \ldots \ldots \ldots \ldots$ & Confirmed & 15, 43, Paper III & $\mathrm{WD}+\mathrm{dM} 3$ & 0.124 & IP & Paper III & $\ldots$ \\
\hline & & & $\mathrm{dM} 0-2$ & $\lesssim 4$ & IP & This work & $\ldots$ \\
\hline $0248+601 \ldots \ldots \ldots \ldots . .$. & Candidate & This work & $\mathrm{dM0}-2$ & $\lesssim 4$ & IP & This work & $\ldots$ \\
\hline J0254-053 ........... & Confirmed & 43,69 & $\mathrm{DA}+\mathrm{K} 0 \mathrm{IV}$ & $\ldots$ & $\mathrm{S}$ & 69 & See Appendix \\
\hline & & & $\mathrm{DA}+\mathrm{K} 0 \mathrm{IV}-\mathrm{III}$ & $\lesssim 0.08$ & IP & 2 & $\ldots$ \\
\hline & & & dK0-4 (G5-K0 III) & $\lesssim 4$ & IP & This work & $\ldots$ \\
\hline $0255+009.2 \ldots \ldots \ldots$ & Confirmed & 51,60 & $\mathrm{DA}+\mathrm{dM} 3 \mathrm{e}$ & Close & $\mathrm{S}$ & 60 & See Appendix \\
\hline & & & $\mathrm{dM}$ or $\mathrm{M}$ III & $\lesssim 4$ & IP & This work & $\ldots$ \\
\hline $0258+184 \ldots \ldots \ldots \ldots \ldots$ & Tentative & 38 , this work & $\ldots$ & $\lesssim 4$ & IP & This work & See Appendix \\
\hline $0303-007 \ldots \ldots \ldots \ldots$ & Confirmed & 43, Papers I and III, 60 & $\mathrm{DA}+\mathrm{dM} 2$ & Close & $\mathrm{S}$ & 75,60 & $\ldots$ \\
\hline & & & WD+dM4 & $\lesssim 0.025$ & IP & Paper III & $\ldots$ \\
\hline & & & $\mathrm{dM} 1-3$ & $\lesssim 4$ & IP & This work & $\cdots$ \\
\hline $0302+621 \ldots \ldots \ldots \ldots . .$. & Tentative & This work & $\ldots$ & $\lesssim 4$ & IP & This work & See Appendix \\
\hline $0308+096 \ldots \ldots \ldots \ldots . . .$. & Confirmed & $8,15,43,54$ & $\mathrm{DA} 2+\mathrm{dM} 4.5$ & Close & IP & 8 & See Appendix \\
\hline & & & $\mathrm{dM} 1-4$ & $\lesssim 4$ & IP & This work & $\cdots$ \\
\hline $0309-275 \ldots \ldots \ldots \ldots$ & Candidate & Paper I & dK7-M0 & $\lesssim 4$ & IP & This work & $\cdots$ \\
\hline $0312+019 \ldots \ldots \ldots \ldots$ & Candidate & This work & $\mathrm{dK} 5-\mathrm{M} 5$ & $\lesssim 4$ & IP & This work & See Appendix \\
\hline $0347-137 \ldots \ldots \ldots \ldots$ & Confirmed & 8, Papers I and III & WD+dM4.5 & 1.052 & IP & Paper III & $\ldots$ \\
\hline & & & $\mathrm{DA} 2+\mathrm{dM} 3$ & $\leq 0.5$ & IP & 8 & $\ldots$ \\
\hline & & & $\mathrm{dM} 1-3$ & $\lesssim 4$ & IP & This work & $\ldots$ \\
\hline J0357+286 ............. & Confirmed & $26,27,43$, Paper I & $\mathrm{WD}+\mathrm{dK} 2$ & Close & $\mathrm{S}$ & 26,27 & See Appendix \\
\hline & & & $\mathrm{dK} 4-\mathrm{dM} 2$ & $\lesssim 4$ & IP & This work & $\ldots$ \\
\hline $0354+463 \ldots \ldots \ldots \ldots . . .$. & Confirmed & 8, 43, Paper III & $\mathrm{WD}+\mathrm{dM} 7$ & $\lesssim 0.025$ & IP & Paper III & $\ldots$ \\
\hline & & & DA6+dM7 & $\leq 0.5$ & IP & 8 & $\ldots$ \\
\hline & & & $\mathrm{dM} 4-5.5$ & $\lesssim 4$ & IP & This work & $\cdots$ \\
\hline $0357-233 \ldots \ldots \ldots \ldots$ & Confirmed & 8, Papers I and III & $\mathrm{WD}+\mathrm{dM} 3$ & 1.190 & IP & Paper III & $\ldots$ \\
\hline & & & $\mathrm{DA} 1+\mathrm{dM} 3$ & 1.2 & IP & 8 & $\ldots$ \\
\hline & & & $\cdots$ & $\lesssim 4$ & IP & This work & $\ldots$ \\
\hline $0357+081 \ldots \ldots \ldots \ldots$ & Tentative & This work & $\ldots$ & $\lesssim 4$ & IP & This work & $\ldots$ \\
\hline
\end{tabular}


TABLE 2-Continued

\begin{tabular}{|c|c|c|c|c|c|c|c|}
\hline $\begin{array}{l}\text { WD } \\
(1)\end{array}$ & $\begin{array}{c}\text { Binary Status } \\
\text { (2) }\end{array}$ & $\begin{array}{c}\text { Binary Status References } \\
\text { (3) }\end{array}$ & $\begin{array}{l}\text { Spectral Types } \\
\text { (4) }\end{array}$ & $\begin{array}{c}a_{\text {sep }} \\
(\operatorname{arcsec}) \\
(5)\end{array}$ & $\begin{array}{l}\text { Data } \\
(6)\end{array}$ & $\begin{array}{c}\text { References } \\
\text { for Cols. (4)-(6) } \\
\text { (7) }\end{array}$ & $\begin{array}{l}\text { Comments } \\
(8)\end{array}$ \\
\hline $0413-077 \ldots \ldots \ldots . .$. & Confirmed & 43, Paper I & $\mathrm{WD}+\mathrm{dM} 4.5 \mathrm{e}$ & $\approx 7$ & I & 68,9 & See Appendix \\
\hline $0416+272 \ldots \ldots \ldots .$. & Tentative & This work & & $\lesssim 4$ & IP & This work & See Appendix \\
\hline \multirow[t]{3}{*}{$0419-487 \ldots \ldots \ldots . .}$. & Confirmed & 3,42 & WD+dM6 & Close & $\mathrm{SP}$ & 3 & See Appendix \\
\hline & & & $\mathrm{WD}+\mathrm{dM} 4$ & Close & $\mathrm{S}$ & 42 & $\ldots$ \\
\hline & & & dM3-4.5 & $\lesssim 4$ & IP & This work & $\ldots$ \\
\hline \multirow[t]{2}{*}{$0429+176 \ldots \ldots \ldots$} & Confirmed & 35,43 , Paper I & $\mathrm{DA}+\mathrm{dM} 4.5 \mathrm{e}$ & Close & SP & 35 & See Appendix \\
\hline & & & $\mathrm{dM} 2-3$ & $\lesssim 4$ & IP & This work & $\ldots$ \\
\hline \multirow[t]{2}{*}{$0430+136 \ldots \ldots \ldots . .}$. & Confirmed & 43,75 , Paper I & $\mathrm{DA}+\mathrm{dM}$ & $\ldots$ & $\mathrm{S}$ & 75 & $\ldots$ \\
\hline & & & $\mathrm{dM} 0.5-2.5$ & $\lesssim 4$ & IP & This work & $\ldots$ \\
\hline \multirow[t]{2}{*}{$0458-662 \ldots \ldots \ldots$} & Confirmed & 24, 43, Papers I and III & $\mathrm{DA}+\mathrm{dM} 2$ & Close & PS & 24 & See Appendix \\
\hline & & & $\mathrm{WD}+\mathrm{dM} 2.5$ & $\lesssim 0.025$ & IP & Paper III & $\ldots$ \\
\hline $0627+299 \ldots \ldots \ldots .$. & Tentative & This work & $\ldots$ & $\lesssim 4$ & IP & This work & See Appendix \\
\hline \multirow{2}{*}{$0628-020 \ldots \ldots \ldots$} & Confirmed & 43, 47, 62, Paper I & $\mathrm{WD}+\mathrm{dM}$ & 4 & IS & 47 & See Appendix \\
\hline & & & $\mathrm{dM} 2.5-4.5$ & $\lesssim 4$ & IP & This work & $\ldots$ \\
\hline $0710+741 \ldots \ldots \ldots . .$. & Confirmed & 8, Paper I & $\mathrm{DA} 3+\mathrm{dM} 7$ & Close & IP & 8 & See Appendix \\
\hline $0718-316 \ldots \ldots \ldots$. & Confirmed & 43,70 & $\mathrm{DAO}+\mathrm{dM} 0-2$ & Close & $\mathrm{S}$ & 70 & See Appendix \\
\hline $0752-146 \ldots \ldots \ldots .$. & Confirmed & 8, Paper I & DA3+dM6 & Close & IP & 8 & $\ldots$ \\
\hline \multirow[t]{2}{*}{$0800-533 \ldots \ldots \ldots . . . . .}$. & Candidate & 77,29 , this work & $\mathrm{dM} 3-4$ & $\ldots$ & $\mathrm{P}$ & 29 & See Appendix \\
\hline & & & $\mathrm{dK} 3-5$ or $\mathrm{dM} 2-3$ & $\lesssim 4$ & IP & This work & 11 \\
\hline $0802+387 \ldots \ldots \ldots . .$. & Tentative & This work & $\ldots$ & $\lesssim 4$ & IP & This work & See Appendix \\
\hline \multirow[t]{2}{*}{$0805+654 \ldots \ldots \ldots \ldots$} & Confirmed & 36 & $\mathrm{WD}+\mathrm{dM}$ & $\ldots$ & $\mathrm{S}$ & 36 & $\ldots$ \\
\hline & & & $\mathrm{dM} 2-4$ & $\lesssim 4$ & IP & This work & $\ldots$ \\
\hline $0812+478 \ldots \ldots \ldots . .$. & Candidate & Paper I & $\ldots$ & $\lesssim 4$ & IP & This work & $\ldots$ \\
\hline \multirow[t]{2}{*}{$0824+288 \ldots \ldots \ldots}$. & Confirmed & $8,15,36,43$ & $\mathrm{DA} 1+\mathrm{dC}+\mathrm{dM} 3.5$ & $\leq 0.5,3.3$ & IP & 8 & $\ldots$ \\
\hline & & & $\mathrm{dK} 6-\mathrm{M} 1$ & $\lesssim 4$ & IP & This work & $\ldots$ \\
\hline $0852+630 \ldots \ldots \ldots .$. & Candidate & This work & $\ldots$ & $\lesssim 4$ & IP & This work & See Appendix \\
\hline \multirow[t]{2}{*}{$0858-220 \ldots \ldots \ldots . .}$. & Confirmed & $43,55,61$ & $\mathrm{DC}+\mathrm{dM}$ & 5 & IPS & 55 & See Appendix \\
\hline & & & $\mathrm{dK} 5-\mathrm{M} 2$ & $\sim 4$ & IP & This work & 11 \\
\hline $0908+226 \ldots \ldots \ldots$. & Candidate & 43, Paper I & late $\mathrm{dK}$ & $\lesssim 4$ & IP & This work & See Appendix \\
\hline $0915+201 \ldots \ldots \ldots . . . . . .$. & Confirmed & Paper I, this work & Mid dK - late dM & $\lesssim 4$ & IP & This work & See Appendix \\
\hline \multirow[t]{2}{*}{$0928+399 \ldots \ldots \ldots$} & Confirmed & 60 & $\mathrm{DA}+\mathrm{dM} 3 \mathrm{e}$ & Close & $\mathrm{S}$ & 60 & $\ldots$ \\
\hline & & & $\mathrm{dM} 3-8$ & $\lesssim 4$ & IP & This work & $\ldots$ \\
\hline \multirow[t]{2}{*}{$0933+025 \ldots \ldots \ldots . .}$. & Confirmed & $8,15,36,43$ & $\mathrm{DA} 2+\mathrm{dM} 3.5$ & $\leq 0.5$ & IP & 8 & $\ldots$ \\
\hline & & & $\mathrm{dM} 2-3$ & $\lesssim 4$ & IP & This work & $\ldots$ \\
\hline $0937-095 \ldots \ldots \ldots . . .$. & Candidate & Paper I & $\mathrm{dK} 4-\mathrm{M} 4$ & $\lesssim 4$ & IP & This work & See Appendix \\
\hline \multirow[t]{2}{*}{$0949+451 \ldots \ldots \ldots . .}$. & Confirmed & Paper III & $\mathrm{WD}+\mathrm{dM} 4.5$ & 2.892 & IP & Paper III & See Appendix \\
\hline & & & $\mathrm{dM} 3-4.5$ & $\lesssim 4$ & IP & This work & $\ldots$ \\
\hline $0950+185 \ldots \ldots \ldots . .$. & Confirmed & $8,15,36,43$ & $\mathrm{DA} 2+\mathrm{dM} 2$ & 1.1 & IP & 8 & $\ldots$ \\
\hline & & & dM0-1 & $\lesssim 4$ & IP & This work & $\ldots$ \\
\hline $0956+045 \ldots \ldots \ldots . .$. & Confirmed & $8,15,43$ & DA3+dM4.5 & 2.0 & IP & 8 & $\ldots$ \\
\hline & & & $\mathrm{dM} 2-5.5$ & $\lesssim 4$ & IP & This work & $\ldots$ \\
\hline $1001+203 \ldots \ldots \ldots$. & Confirmed & $8,15,36,43$, Paper I & $\mathrm{DA} 2+\mathrm{dM} 3$ & $\leq 0.5$ & IP & 8 & $\cdots$ \\
\hline & & & $\mathrm{dM} 1-3.5$ & $\lesssim 4$ & IP & This work & $\ldots$ \\
\hline $1004-178 \ldots \ldots \ldots . .$. & Candidate & This work & $\mathrm{dM} 0.5-3$ & $\lesssim 4$ & IP & This work & $\ldots$ \\
\hline $1013-050 \ldots \ldots \ldots . .$. & Confirmed & 8,43 , Paper I & $\mathrm{DAO} 1+\mathrm{dM} 4.5+\mathrm{dM} 1+\mathrm{dM} 1$ & Close, $3.2,3.2$ & IP & 8 & See Appendix \\
\hline & & & $\mathrm{dM} 0-2.5$ & $\lesssim 4$ & IP & This work & $\ldots$ \\
\hline $1015-173 \ldots \ldots \ldots$ & Candidate & Paper I & $\ldots$ & $\lesssim 4$ & IP & This work & $\ldots$ \\
\hline $1026+002 \ldots \ldots \ldots . .$. & Confirmed & $8,15,36,43,54$, Paper I & $\mathrm{DA} 3+\mathrm{dM} 4 \mathrm{e}$ & Close & $\mathrm{S}$ & 54 & See Appendix \\
\hline & & & $\mathrm{DA} 3+\mathrm{dM} 4.5$ & Close & IP & 8 & $\ldots$ \\
\hline & & & $\mathrm{dM} 2.5-4$ & $\lesssim 4$ & IP & This work & $\ldots$ \\
\hline $1027-039 \ldots \ldots \ldots$. & Candidate & This work & $\mathrm{dM} 2-7$ & $\lesssim 4$ & IP & This work & See Appendix \\
\hline $1033+464 \ldots \ldots \ldots . .$. & Confirmed & $8,14,15,36,43$ & $\mathrm{DA} 2+\mathrm{dM} 4.5$ & $\leq 0.5$ & IP & 8 & $\ldots$ \\
\hline & & & $\mathrm{dM} 2.5-4$ & $\lesssim 4$ & IP & This work & $\ldots$ \\
\hline & & & $\mathrm{WD}+\mathrm{dM} 4-5$ & $\lesssim 10$ & IP & 14 & $\ldots$ \\
\hline $1036-204 \ldots \ldots \ldots . .$. & Tentative & Paper II, this work & & $\lesssim 4$ & IP & This work & See Appendix \\
\hline $1037+512 \ldots \ldots \ldots . .$. & Confirmed & 36, Paper I & $\mathrm{WD}+\mathrm{dM}$ & $\ldots$ & $\mathrm{S}$ & 36 & $\ldots$ \\
\hline & & & $\mathrm{dM} 2.5-4.5$ & $\lesssim 4$ & IP & This work & $\ldots$ \\
\hline $1042-690 \ldots \ldots \ldots$. & Confirmed & 8,44 & $\mathrm{DA} 2+\mathrm{dM} 4.5$ & Close & IP & 8 & See Appendix \\
\hline & & & $\mathrm{dM} 4-5$ & $\lesssim 4$ & IP & This work & . \\
\hline $1049+103 \ldots \ldots \ldots . .$. & Confirmed & $8,15,36,43$ & $\mathrm{DA} 2+\mathrm{dM} 4$ & $\leq 0.5$ & IP & 8 & $\ldots$ \\
\hline & & & $\ldots$ & $\lesssim 4$ & IP & This work & $\ldots$ \\
\hline $1055-072 \ldots \ldots \ldots$. & Tentative & This work & $\ldots$ & $\lesssim 4$ & IP & This work & See Appendix \\
\hline $1101+364 \ldots \ldots \ldots \ldots$ & Tentative & This work & $\ldots$ & $\lesssim 4$ & IP & This work & See Appendix \\
\hline $1104+044 \ldots \ldots \ldots . .$. & Confirmed & This work & $\mathrm{WD}+\mathrm{dK} 0-4$ & $\approx 3$ & IP & This work & See Appendix \\
\hline $1106+316 \ldots \ldots \ldots$ & Candidate & Paper I & dK5-M0 & $\lesssim 4$ & IP & This work & . \\
\hline $1106-211 \ldots \ldots \ldots . .$. & Tentative & Paper I & $\ldots$ & $\lesssim 4$ & IP & This work & See Appendix \\
\hline
\end{tabular}


TABLE 2-Continued

\begin{tabular}{|c|c|c|c|c|c|c|c|}
\hline $\begin{array}{l}\text { WD } \\
(1)\end{array}$ & $\begin{array}{c}\text { Binary Status } \\
\text { (2) }\end{array}$ & $\begin{array}{c}\text { Binary Status References } \\
\text { (3) }\end{array}$ & $\begin{array}{c}\text { Spectral Types } \\
\text { (4) }\end{array}$ & $\begin{array}{c}a_{\text {sep }} \\
(\operatorname{arcsec}) \\
(5)\end{array}$ & $\begin{array}{l}\text { Data } \\
(6)\end{array}$ & $\begin{array}{c}\text { References } \\
\text { for Cols. (4)-(6) } \\
\text { (7) }\end{array}$ & $\begin{array}{c}\text { Comments } \\
\text { (8) }\end{array}$ \\
\hline \multirow[t]{3}{*}{$1123+189 \ldots \ldots \ldots \ldots \ldots$} & Confirmed & $8,15,43,56$, Paper I & $\mathrm{DA} 1+\mathrm{dM} 3$ & 1.3 & IP & 8 & $\ldots$ \\
\hline & & & $\mathrm{dM} 2-3$ & $\lesssim 4$ & IP & This work & $\ldots$ \\
\hline & & & $\mathrm{DA} 4+\mathrm{dM}$ & $\ldots$ & $\mathrm{S}$ & 56 & $\ldots$ \\
\hline \multirow[t]{2}{*}{$1126+185 \ldots \ldots \ldots \ldots \ldots \ldots$} & Tentative & 15,49 & $\mathrm{DC} 8+\mathrm{dG}-\mathrm{K}$ & $\ldots$ & $\mathrm{S}$ & 49 & See Appendix \\
\hline & & & $\mathrm{dK} 1-4$ & $\lesssim 4$ & IP & This work & $\ldots$ \\
\hline \multirow{2}{*}{$1132-298 \ldots \ldots \ldots \ldots \ldots \ldots$} & Confirmed & 43,53 & $\mathrm{DA}+\mathrm{dM} 4$ & Close & $\mathrm{S}$ & 53 & See Appendix \\
\hline & & & $\mathrm{dK} 7-\mathrm{M} 2$ & $\lesssim 4$ & IP & This work & $\ldots$ \\
\hline \multirow[t]{2}{*}{$1133+358 \ldots \ldots \ldots \ldots \ldots$} & Confirmed & 17, 43, 49, Paper I & $\mathrm{DC}+\mathrm{dM} 4.5 \mathrm{e}$ & $\ldots$ & $\mathrm{S}$ & 49 & $\ldots$ \\
\hline & & & $\mathrm{dM} 2.5-4$ & $\lesssim 4$ & IP & This work & $\ldots$ \\
\hline \multirow[t]{3}{*}{$1136+667 \ldots \ldots \ldots \ldots \ldots \ldots$} & Confirmed & $21,23,43,63$, Paper I & $\mathrm{DAO}+\mathrm{dMe}$ & Close & $\mathrm{S}$ & 23 & See Appendix \\
\hline & & & $\mathrm{DAO}+\mathrm{dK} 7$ & Close & $\mathrm{S}$ & 63 & $\ldots$ \\
\hline & & & dK6-M0 & $\lesssim 4$ & IP & This work & $\ldots$ \\
\hline \multirow{2}{*}{$1141+504 \ldots \ldots \ldots \ldots \ldots \ldots$} & Confirmed & 15,60 & $\mathrm{DA}+\mathrm{dM} 4 \mathrm{e}$ & Close & $\mathrm{S}$ & 60 & $\ldots$ \\
\hline & & & dK3-M3.5 & $\lesssim 4$ & IP & This work & $\ldots$ \\
\hline $1147+371 \ldots \ldots \ldots \ldots \ldots \ldots$ & Candidate & This work & $\mathrm{dK} 5-\mathrm{M} 2.5$ & $\lesssim 4$ & IP & This work & $\cdots$ \\
\hline $1156+129 \ldots \ldots \ldots \ldots \ldots$ & Tentative & Paper I & dK6-M2.5 & $\lesssim 4$ & IP & This work & See Appendix \\
\hline \multirow{2}{*}{ 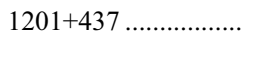 } & Tentative & $12,15,43$, Paper I & $\mathrm{DC}+\mathrm{dMe}$ & $\ldots$ & $\ldots$ & 12 & See Appendix \\
\hline & & & $\ldots$ & $\lesssim 4$ & IP & This work & $\ldots$ \\
\hline \multirow{2}{*}{$1210+464 \ldots \ldots \ldots \ldots \ldots$} & Confirmed & $8,15,36,43$, Paper I & $\mathrm{DA} 2+\mathrm{dM} 2$ & $\leq 0.5$ & IP & 8 & $\ldots$ \\
\hline & & & $\mathrm{dM} 0-2$ & $\lesssim 4$ & IP & This work & $\ldots$ \\
\hline \multirow[t]{3}{*}{$1213+528 \ldots \ldots \ldots \ldots \ldots \ldots$} & Confirmed & $34,43,50$ & $\mathrm{DA}+\mathrm{dM} 2$ & Close & $\mathrm{S}$ & 34 & See Appendix \\
\hline & & & $\mathrm{dM} 3-5$ & $\lesssim 4$ & IP & This work & $\ldots$ \\
\hline & & & $\mathrm{DA}+\mathrm{dM} 4.5 \mathrm{e}$ & $\ldots$ & $\mathrm{P}$ & 50 & $\ldots$ \\
\hline \multirow[t]{2}{*}{$1214+032 \ldots \ldots \ldots \ldots \ldots \ldots$} & Confirmed & $43,55,62$, Paper I & $\mathrm{DA}+\mathrm{sdM} 3$ & 2 & IS & 55,62 & $\ldots$ \\
\hline & & & $\mathrm{dM} 2-3$ & $\lesssim 4$ & IP & This work & $\ldots$ \\
\hline \multirow[t]{3}{*}{$1218+497 \ldots \ldots \ldots \ldots \ldots$} & Confirmed & 60, Papers I and III & $\mathrm{WD}+\mathrm{dM} 4$ & 0.302 & IP & Paper III & $\ldots$ \\
\hline & & & $\mathrm{DA}+\mathrm{dM} 4 \mathrm{e}$ & Close & $\mathrm{S}$ & 60 & $\ldots$ \\
\hline & & & $\mathrm{dK} 4-\mathrm{M} 2$ & $\lesssim 4$ & IP & This work & $\ldots$ \\
\hline $1224+309 \ldots \ldots \ldots \ldots \ldots$ & Confirmed & 15, 46, Paper I & $\mathrm{DA}+\mathrm{dM} 4+$ & Close & PS & 46 & See Appendix \\
\hline 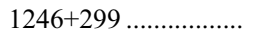 & Candidate & This work & $\mathrm{dK} 3-\mathrm{M} 0$ & $\lesssim 4$ & IP & This work & $\ldots$ \\
\hline \multirow[t]{2}{*}{$1247-176 \ldots \ldots \ldots \ldots \ldots$} & Confirmed & 32, 33, Paper I & $\mathrm{DA}+\mathrm{dMe}$ & Close & $\mathrm{S}$ & 32,33 & See Appendix \\
\hline & & & $\mathrm{dM} 1-3$ & $\lesssim 4$ & IP & This work & $\ldots$ \\
\hline $\mathrm{J} 1255+258 \ldots \ldots \ldots \ldots \ldots$ & Confirmed & 10,43 & $\mathrm{sdO}+\mathrm{G} 5 \mathrm{III}$ & Close & $\mathrm{S}$ & 10,25 & See Appendix \\
\hline & & & $\ldots$ & $\lesssim 0.05$ & I & 5 & $\ldots$ \\
\hline & & & G3-K0 III & $\lesssim 4$ & IP & This work & $\ldots$ \\
\hline $1254-133 \ldots \ldots \ldots \ldots \ldots$ & Candidate & This work & $\mathrm{dM} 0-5$ & $\lesssim 4$ & IP & This work & $\ldots$ \\
\hline 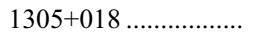 & Confirmed & 4 , this work & $\mathrm{dM} 1-3.5$ & $\lesssim 2$ & IP & This work & See Appendix \\
\hline $1307-141 \ldots \ldots \ldots \ldots \ldots$ & Candidate & Paper I & $\mathrm{dK} 7-\mathrm{M} 2.5$ & $\lesssim 4$ & IP & This work & $\ldots$ \\
\hline $1310-305 \ldots \ldots \ldots \ldots \ldots$ & Tentative & This work & $\ldots$ & $\lesssim 4$ & IP & This work & See Appendix \\
\hline 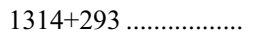 & Confirmed & 36,39 & $\mathrm{DAwk}+\mathrm{dM} 3.5 \mathrm{e}$ & 3 & IPS & 39 & See Appendix \\
\hline & & & $\mathrm{dM} 0.5-3$ & $\lesssim 4$ & IP & This work & $\ldots$ \\
\hline $1319-288 \ldots \ldots \ldots \ldots \ldots$ & Confirmed & 33 & $\mathrm{DA}+\mathrm{dM}$ & $\ldots$ & $\mathrm{S}$ & 33 & $\ldots$ \\
\hline 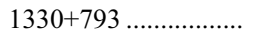 & Confirmed & Paper I & $\mathrm{dM} 0-2$ & $\approx 3.9$ & IP & This work & See Appendix \\
\hline $1333+487 \ldots \ldots \ldots \ldots \ldots . .$. & Confirmed & 16, 43, Papers I and III & WD+dM5 & 2.947 & IP & Paper III & $\ldots$ \\
\hline & & & $\mathrm{DB}+\mathrm{dM}$ & "Unresolved visually" & PS & 16 & $\ldots$ \\
\hline & & & $\mathrm{dM} 2-4$ & $\lesssim 4$ & IP & This work & $\ldots$ \\
\hline $\mathrm{J} 1340+604 \ldots \ldots \ldots \ldots \ldots$ & Confirmed & 43, 60, Paper III & WD+dM4 & $\lesssim 0.025$ & IP & Paper III & See Appendix \\
\hline & & & $\mathrm{DA}+\mathrm{dM} 3 \mathrm{e}$ & Close & $\mathrm{S}$ & 60 & $\ldots$ \\
\hline $1401+005 \ldots \ldots \ldots \ldots \ldots$ & Candidate & This work & $\mathrm{dK} 5+$ & $\lesssim 4$ & IP & This work & $\ldots$ \\
\hline $1412-049 \ldots \ldots \ldots \ldots \ldots$ & Confirmed & Papers I and III & $\mathrm{WD}+\mathrm{dM} 0$ & 3.508 & IP & Paper III & $\ldots$ \\
\hline $1412-109$................... & Confirmed & This work & K8-M5 III & $\lesssim 4$ & IP & This work & See Appendix \\
\hline $1415+132 \ldots \ldots \ldots \ldots \ldots . . .$. & Confirmed & 11,19 & $\mathrm{DA}+\mathrm{dM} 3+$ & Close & $\mathrm{S}$ & 11 & $\ldots$ \\
\hline & & & $\mathrm{dK} 3-\mathrm{M} 2$ & $\lesssim 4$ & IP & This work & $\ldots$ \\
\hline $1424+503 \ldots \ldots \ldots \ldots \ldots$ & Confirmed & 43,59 & $\mathrm{DA} 2+\mathrm{dM}$ & Close & $\mathrm{S}$ & 59 & See Appendix \\
\hline & & & $\mathrm{dK} 7-\mathrm{M} 2.5$ & $\lesssim 4$ & IP & This work & $\ldots$ \\
\hline $1433+538 \ldots \ldots \ldots \ldots \ldots$ & Confirmed & $15,43,60$, Paper III & $\mathrm{DA}+\mathrm{dM} 4$ & Close & $\mathrm{S}$ & 60 & See Appendix \\
\hline & & & WD+dM5 & $\lesssim 0.025$ & IP & Paper III & $\ldots$ \\
\hline & & & $\mathrm{DA} 2+\mathrm{dM} 4.5$ & $\leq 0.5$ & IP & 8 & $\ldots$ \\
\hline $1435+370 \ldots \ldots \ldots \ldots \ldots . .$. & Confirmed & Papers I and III & $\mathrm{WD}+\mathrm{dM} 2.5$ & 1.251 & IP & Paper III & $\ldots$ \\
\hline $1436-216 \ldots \ldots \ldots \ldots \ldots$ & Confirmed & 78, Paper I & $\mathrm{DA}+\mathrm{dM}$ & $\ldots$ & $\mathrm{S}$ & 78 & $\ldots$ \\
\hline & & & $\mathrm{dM} 2-3.5$ & $\lesssim 4$ & IP & This work & $\ldots$ \\
\hline $1443+336 \ldots \ldots \ldots \ldots \ldots . . .$. & Confirmed & 15, 36, Papers I and III & $\mathrm{WD}+\mathrm{dM} 2.5$ & 0.679 & IP & Paper III & See Appendix \\
\hline & & & $\mathrm{dK} 4-\mathrm{M} 3$ & $\lesssim 4$ & IP & This work & $\ldots$ \\
\hline $1458+171 \ldots \ldots \ldots \ldots \ldots . . .$. & Confirmed & Papers I and III & WD+dM5 & $\lesssim 0.025$ & IP & Paper III & $\ldots$ \\
\hline & & & $\mathrm{dM} 4-5.5$ & $\lesssim 4$ & IP & This work & $\ldots$ \\
\hline & & & $\mathrm{dM} 4-5.5$ & $\lesssim 4$ & IP & This work & $\ldots$ \\
\hline
\end{tabular}




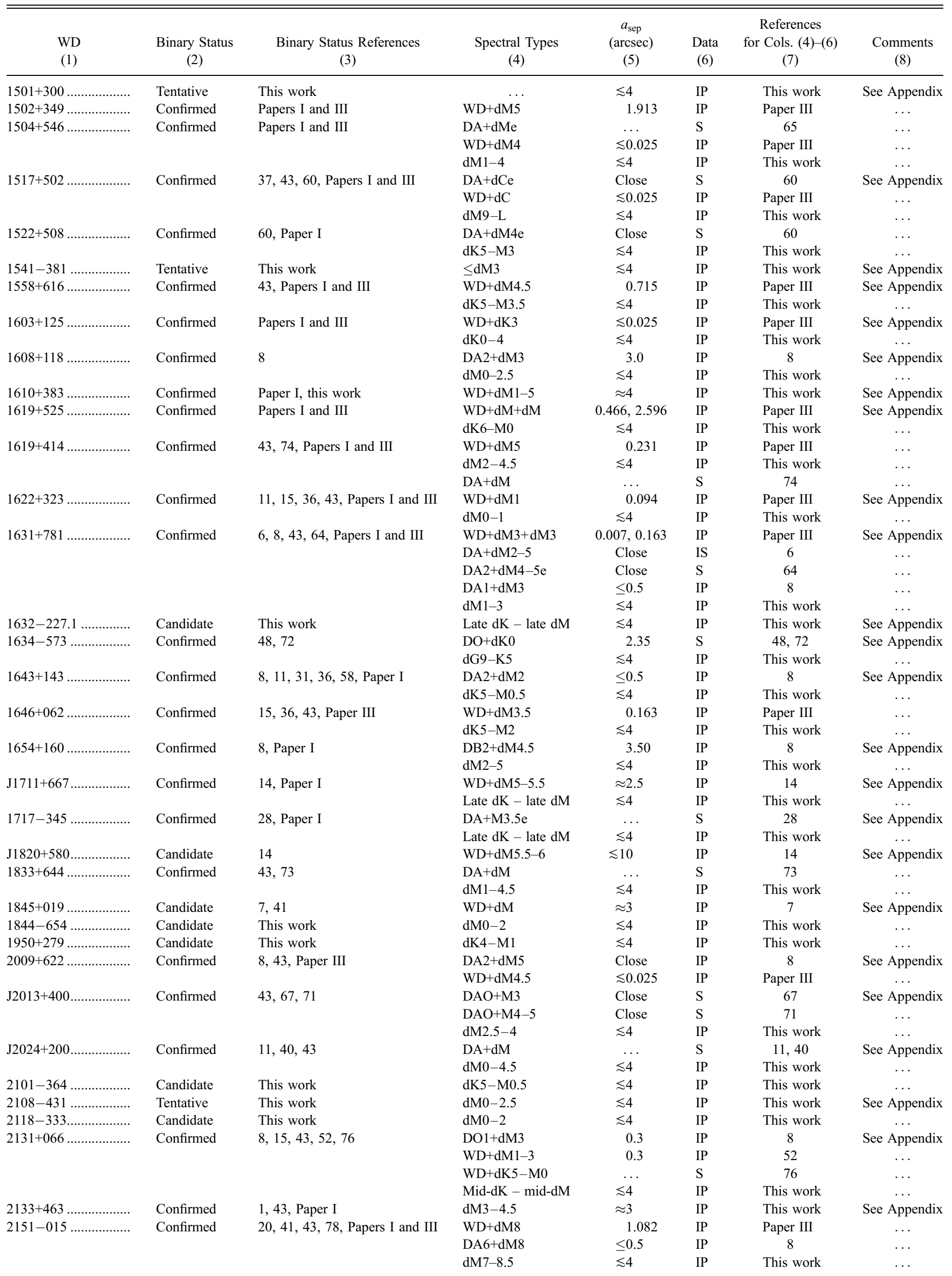


TABLE 2-Continued

\begin{tabular}{|c|c|c|c|c|c|c|c|}
\hline $\begin{array}{l}\text { WD } \\
\text { (1) }\end{array}$ & $\begin{array}{c}\text { Binary Status } \\
\text { (2) }\end{array}$ & $\begin{array}{c}\text { Binary Status References } \\
\text { (3) }\end{array}$ & $\begin{array}{c}\text { Spectral Types } \\
\text { (4) }\end{array}$ & $\begin{array}{c}a_{\mathrm{sep}} \\
(\operatorname{arcsec}) \\
(5)\end{array}$ & $\begin{array}{l}\text { Data } \\
(6)\end{array}$ & $\begin{array}{c}\text { References } \\
\text { for Cols. (4)-(6) } \\
\text { (7) }\end{array}$ & $\begin{array}{l}\text { Comments } \\
(8)\end{array}$ \\
\hline $2154+408 \ldots \ldots \ldots \ldots \ldots \ldots \ldots$ & Confirmed & 8,22 & $\mathrm{DA} 2+\mathrm{dM} 3.5$ & Close & IP & 8 & See Appendix \\
\hline $2237+819 \ldots \ldots \ldots \ldots \ldots \ldots$ & Confirmed & 13,43 & $\begin{array}{l}\text { WD+dM3-4 } \\
\text { dM3-5 }\end{array}$ & $\begin{array}{l}\text { Close } \\
\lesssim 4\end{array}$ & $\begin{array}{l}\text { PS } \\
\text { IP }\end{array}$ & $\begin{array}{l}13 \\
\text { This work }\end{array}$ & $\begin{array}{l}\text { See Appendix } \\
\ldots\end{array}$ \\
\hline $2256+249 \ldots \ldots \ldots \ldots \ldots \ldots$ & Confirmed & $8,43,57$, Paper I & $\begin{array}{l}\mathrm{DA} 2+\mathrm{dM} 4 \\
\mathrm{DA} 2+\mathrm{M} 3-5\end{array}$ & $\begin{array}{l}\text { Close } \\
\text { Close }\end{array}$ & $\begin{array}{l}\text { IP } \\
\text { PS }\end{array}$ & $\begin{array}{l}8 \\
57\end{array}$ & $\begin{array}{l}\text { See Appendix } \\
\ldots\end{array}$ \\
\hline $2311-068 \ldots \ldots \ldots \ldots \ldots \ldots$ & Tentative & This work & $\ldots$ & $\lesssim 4$ & IP & This work & See Appendix \\
\hline $2317+268 \ldots \ldots \ldots \ldots \ldots \ldots$ & Confirmed & Papers I and III & $\begin{array}{l}\mathrm{WD}+\mathrm{dM} 3.5 \\
\mathrm{dM} 2-5\end{array}$ & $\begin{array}{l}\lesssim 0.025 \\
\lesssim 4\end{array}$ & $\begin{array}{l}\text { IP } \\
\text { IP }\end{array}$ & $\begin{array}{l}\text { Paper III } \\
\text { This work }\end{array}$ & ${ }^{\cdots}$ \\
\hline $2318-137 \ldots \ldots \ldots \ldots \ldots$ & Tentative & This work & $\mathrm{dM} 1-4$ & $\approx 3$ & IP & This work & See Appendix \\
\hline $2326-224 \ldots \ldots \ldots \ldots \ldots \ldots$ & Confirmed & Paper I, this work & $\mathrm{dM} 1-4$ & $\approx 4$ & IP & This work & See Appendix \\
\hline $2336-187 \ldots \ldots \ldots \ldots$ & Tentative & Paper I & $\ldots$ & $\approx 4$ & IP & This work & See Appendix \\
\hline
\end{tabular}

References.-(1) Bakos et al. 2002; (2) Barstow et al. 2001; (3) Bruch \& Diaz 1998; (4) Cheselka et al. 1993; (5) Ciardullo et al. 1999; (6) Cooke et al. 1992; (7) Debes et al. 2005b; (8) Farihi et al. 2005a; (9) Feibelman 1986; (10) Feibelman \& Kaler 1983; (11) Finley et al. 1997; (12) Fleming et al. 1993; (13) Gänsicke et al. 2004; (14) Green et al. 2000; (15) Green et al. 1986; (16) Greenstein 1975; (17) Greenstein 1976; (18) Greenstein 1984; (19) Greenstein 1986; (20) Greenstein \& Liebert 1990; (21) Heber et al. 1996; (22) Hillwig et al. 2002; (23) Holberg et al. 2001; (24) Hutchings et al. 1995; (25) Jasniewicz et al. 1996; (26) Jeffries et al. 1996; (27) Jeffries \& Smalley 1996; (28) Kawka et al. 2004; (29) Kawka et al. 2007; (30) Kellett et al. 1995; (31) Kidder et al. 1991; (32) Kilkenny et al. 1997; (33) Koester et al. 2001; (34) Lanning 1982; (35) Lanning \& Pesch 1981; (36) Liebert et al. 2005; (37) Liebert et al. 1994; (38) Lisker et al. 2005; (39) Margon et al. 1976; (40) Mason et al. 1995; (41) Maxted \& Marsh 1999; (42) Maxted et al. 2007; (43) McCook \& Sion 1999; (44) Morales-Rueda et al. 2005; (45) Mueller \& Bues 1987; (46) Orosz et al. 1999; (47) Oswalt et al. 1988; (48) Parsons et al. 1976; (49) Putney 1997; (50) Probst 1983; (51) Raymond et al. 2003; (52) Reed et al. 2000; (53) Ruiz \& Maza 1990; (54) Saffer et al. 1993; (55) Salim \& Gould 2003; (56) Schmidt \& Smith 1995; (57) Schmidt et al. 1995; (58) Schultz et al. 1996; (59) Schwartz et al. 1995; (60) Silvestri et al. 2006; (61) Silvestri et al. 2002; (62) Silvestri et al. 2001; (63) Sing et al. 2004; (64) Sion et al. 1995; (65) Stepanian et al. 2001; (66) Thorstensen et al. 1978; (67) Thorstensen et al. 1994; (68) van den Bos 1926; (69) Vennes et al. 1995; (70) Vennes \& Thorstensen 1994; (71) Vennes et al. 1999; (72) Wegner 1979; (73) Wegner \& McMahan 1988; (74) Wegner \& Swanson 1990b; (75) Wegner et al. 1987; (76) Wesemael et al. 1985; (77) Wickramasinghe \& Bessell 1977; (78) Zuckerman et al. 2003; (79) Zwitter \& Munari 1995.

rejected as misidentifications in Paper I) is only 19\% (the remaining $1 \%$, one $\mathrm{WD}$, is, as yet, neither confirmed as a binary nor rejected from the sample). In this work, $69 \%$ of the reported WDs with near-IR excess are recovered from already known or recently confirmed binaries. We conclude that the near-IR color selection method is not only simple to apply, but also has a high success rate and a correspondingly low rate of false positives (most of which are attributable to incorrect target identifications, not erroneous color selection of single WDs).

Of the 15 tentative candidates reported in Paper I, only three remain in the sample reported in this work (with the rest having been excluded for having large uncertainties on their 2MASS photometry). One of these three has been confirmed as a binary, one has been "upgraded" to a firm candidate due to a decrease in the uncertainties of its recalibrated photometry in the All Sky PSC, and the third remains a tentative candidate. Eight of the 12 excluded targets are now believed to be single WDs (e.g., as described in Paper II and Tremblay \& Bergeron 2007). There is a total of 21 tentative candidates reported in this work, 12 of which were selected because they satisfy only the $\left(H-K_{s}\right)$ criterion (i.e., they are the targets identified with the simulated binaries containing the lowest mass companion stars). However, three of the targets selected from only the $\left(H-K_{S}\right)$ criterion are

TABLE 3

White Dwarf Statistics

\begin{tabular}{|c|c|c|c|c|}
\hline \multirow[b]{2}{*}{ Category } & \multicolumn{4}{|c|}{ Status IN This Work } \\
\hline & Total & Confirmed & Candidate & Tentative \\
\hline This work totals.. & 153 & 105 & 27 & 21 \\
\hline New to this work & 82 & 46 & 19 & 17 \\
\hline Candidate in Paper $\mathrm{I}^{\mathrm{a}}$.. & 68 & 58 & 7 & 3 \\
\hline Tentative in Paper $\mathrm{I}^{\mathrm{b}}$................. & 3 & 1 & 1 & 1 \\
\hline
\end{tabular}

\footnotetext{
${ }^{\text {a }}$ From Table 1 in Paper I.

${ }^{b}$ From Table 2 in Paper I.
}

confirmed binaries (with the remainder of the $H-K_{s}$-selected objects in the candidate class). So, in this regard, we note that it is important when using any method of selecting binary candidates to heed the warning implied by the label tentative candidate, but that rejecting these systems outright would result in the loss of a not-insignificant fraction of true binaries. ${ }^{9}$

\subsubsection{Where Are the Binaries Containing Brown Dwarfs?}

Since the near-IR color-color diagram shown in Figures 1 and 2 contains over $60 \%$ more red-excess WDs than the colorcolor diagram from Paper I, a pertinent question is: why does the current color-color diagram not look more like the simulated diagram from Paper I? Put another way, where are the binaries containing a WD and a M5+ or L-type (potential brown dwarf) companion? Discounting WD $1517+502$ (see $\S 2.3 .1$ ), there are no observed candidates in the L spectral type region of the color-color diagram. Only eight targets in total (again excluding the two objects with anomalous colors; see $\S 2.3 .1)$ have nominal colors redward of the $\left(H-K_{s}\right)$ color of a dM5 star, although several times this many have colors within $1 \sigma$ of this boundary (Figs. 1 and 2, vertical dashed line). Of the confirmed binaries in our sample, only six have estimated spectral types for the companion later than M5 (WD 0145-221, WD 0354+463, WD 0419487, WD 0710+741, WD 0752-146, and WD 2151-015; see Table 2 and Fig. 2). Of these, only WD $0145-221$ has an L-type

\footnotetext{
${ }^{9}$ Incidentally, WD $0518+333$, which was identified as a tentative binary candidate in Paper I and is reported by Tremblay \& Bergeron (2007) as a single WD, is shown by our high angular resolution imaging to have a faint neighbor at a separation of $a \approx 1.9^{\prime \prime}$ in 2005 (from a paper currently in preparation). If the neighbor is associated with the WD, then this is slightly below the expected 2MASS imaging resolution limit. If the neighbor is not associated with the WD (and hence does not share its proper motion), then the separation would have been $a \lesssim 0.5^{\prime \prime}$ at the time of the 2MASS observation in 1998. Thus, this neighbor possibly contaminated the 2MASS photometry. We note that the known bright common proper motion companion to the WD (located $\approx 8^{\prime \prime}$ northeast) is also resolvable into a close $\left(a \approx 0.15^{\prime \prime}\right)$ pair of stars that are approximately equally bright at $I$ band.
} 
TABLE 4

Absolute Magnitudes of Low-Mass Stars

\begin{tabular}{|c|c|c|c|c|c|c|}
\hline \multirow[b]{2}{*}{ SPeCtral TyPe } & \multicolumn{3}{|c|}{ Absolute Magnitude $^{\mathrm{a}}$} & \multicolumn{3}{|c|}{ Maximum Distance Modulus $^{b}$} \\
\hline & $M_{J}$ & $M_{H}$ & $M_{K_{s}}$ & $(m-M)_{J}$ & $(m-M)_{H}$ & $(m-M)_{K_{s}}$ \\
\hline M0 .... & 6.45 & 5.82 & 5.62 & 9.35 & 9.28 & 8.67 \\
\hline 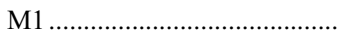 & 6.72 & 6.11 & 5.88 & 9.08 & 8.99 & 8.41 \\
\hline $\mathrm{M} 2$. & 6.98 & 6.38 & 6.14 & 8.82 & 8.72 & 8.16 \\
\hline ................ & 7.24 & 6.68 & 6.40 & 8.56 & 8.42 & 7.90 \\
\hline 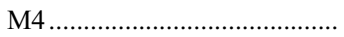 & 8.34 & 7.80 & 7.50 & 7.46 & 7.30 & 6.80 \\
\hline M5 ....... & 9.44 & 8.88 & 8.54 & 6.36 & 6.22 & 5.76 \\
\hline M6 ......... & 10.18 & 9.54 & 9.15 & 5.62 & 5.56 & 5.15 \\
\hline M7 & 10.92 & 10.29 & 9.89 & 4.88 & 4.81 & 4.41 \\
\hline 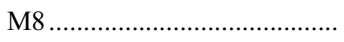 & 11.14 & 10.46 & 10.05 & 4.66 & 4.64 & 4.24 \\
\hline (2) & 11.43 & 10.71 & 10.26 & 4.37 & 4.39 & 4.04 \\
\hline 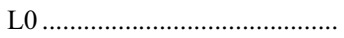 & 11.72 & 10.90 & 10.32 & 4.08 & 4.20 & 3.98 \\
\hline 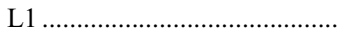 & 12.00 & 11.20 & 10.57 & 3.80 & 3.90 & 3.73 \\
\hline $\mathrm{L} 2 \ldots \ldots$. & 12.29 & 11.28 & 10.58 & 3.51 & 3.82 & 3.72 \\
\hline 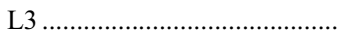 & 12.58 & 11.54 & 10.86 & 3.22 & 3.56 & 3.44 \\
\hline 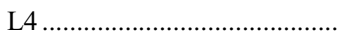 & 12.87 & 11.68 & 11.00 & 2.93 & 3.42 & 3.30 \\
\hline L5 & 13.16 & 11.99 & 11.29 & 2.64 & 3.11 & 3.01 \\
\hline 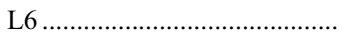 & 14.31 & 13.13 & 12.25 & 1.49 & 1.97 & 2.05 \\
\hline 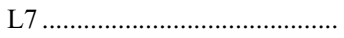 & 14.45 & 13.27 & 12.51 & 1.35 & 1.83 & 1.79 \\
\hline 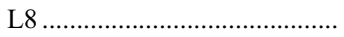 & 14.58 & 13.34 & 12.62 & 1.22 & 1.76 & 1.68 \\
\hline $\mathrm{WD}^{\mathrm{c}}$ & $13.19(57)$ & $13.12(50)$ & $13.06(48)$ & 2.61 & 1.98 & 1.24 \\
\hline
\end{tabular}

${ }^{a}$ From Bessell \& Brett (1988), Gizis et al. (2000), Kirkpatrick et al. (2000), and Hawley et al. (2002).

${ }^{\mathrm{b}}$ For 2MASS good detection limits of $J_{\lim }=15.8, H_{\text {lim }}=15.1$, and $K_{s, \lim }=14.3$.

c Average photometry from the single WD sample used in the Paper I simulation.

companion (L6-7; i.e., a "true" brown dwarf); the rest have companion spectral types of M6-8. However, the near-IR colors of WD 0145-221 are most similar to an early dK star-nowhere near the $\mathrm{L}$ spectral type region in the color-color diagram! This object is at a distance of $d \approx 39 \mathrm{pc}$, so the companion star alone would not have been detected at the good level by 2MASS (see below), and over $90 \%$ of its $J$-band flux is attributed to the WD (Farihi \& Christopher 2004; Farihi et al. 2005b). Although there is an observed excess in both the $H$ and $K_{S}$ bands compared to a single WD model, the observed slope of the spectral energy distribution from $H$ to $K_{s}$ mimics that of the WD component (see Fig. 1 in Farihi et al. 2005b). When combined with the almost complete lack of a $J$-band excess, the true nature of this binary is obfuscated in the color-color diagram. Although color-selected as a binary candidate, the brown dwarf nature of the companion is revealed only through additional analysis. It is possible, then, that some binaries with brown dwarf companions are similarly "hiding" in the near-IR color-color diagram among the total sample of red-excess WDs.

However, we should also explore this issue from the point of view of the assumptions that went into the simulation from Paper I, compared to the real characteristics of the 2MASS survey and the expectations for the presence of WD + brown dwarf binaries. In this context, part of the answer lies in the fact that the simulation in Paper I assumed that all of the simulated binaries are located at $d=10 \mathrm{pc}$ and that all of them are detectable by 2MASS. In fact, most ( $70 \%)$ of the WDs from Table 1 have distance estimates in the literature (e.g., compiled in Paper III; Holberg et al. 2002; Silvestri et al. 2002; Farihi et al. 2005a; Liebert et al. 2005), and we find an average distance of $d=150 \mathrm{pc}$ for our binary candidates, with distances ranging from $\approx 5$ to $700 \mathrm{pc}$ (plus one $7 \sigma$ outlier at $d=1660 \mathrm{pc}$ ). Table 4 lists the absolute magnitudes of late main-sequence spectral types, along with the corresponding maximum distance moduli for detection within the $\mathrm{S} / \mathrm{N} \geq 10$ photometric limits of 2MASS (see $\S 2.1$ ). So, for example, stars later than M5-6 are only detected (at the good level) if they are closer than $d \approx 150 \mathrm{pc}$ (distance modulus of 5.88), while stars later than L6 are only detected if they are closer than $d \approx 25 \mathrm{pc}$ (distance modulus of 1.99).

The table also lists the mean photometry for the sample of single WDs used in the simulation from Paper I. If we take these values as representative, then for a given WD + low-mass star binary, the components will be approximately equally bright in the $J$ and $H$ bands for companion spectral types of L3-5 and L5-6, respectively. The WD never exceeds the $K_{s}$-band brightness of the companion at even the latest $L$ spectral type. In the case of binaries containing equally bright components (in a particular photometric band), the combined absolute magnitude is 0.75 mag brighter than either component alone, allowing for detection out to a factor of $\sqrt{2}$ larger distance. However, as the companion spectral type becomes earlier or later, the combined photometry of the binary is rapidly dominated by the companion or the WD, respectively, and the maximum distance modulus for a good detection in 2MASS rapidly converges back to the singlestar values listed in Table 4. Thus, we should expect that binaries containing late-M or mid-L companions should be detectable at the good level in 2MASS out to distances of $d \approx 150 \mathrm{pc}$ or $d \approx 25 \sqrt{2}=35 \mathrm{pc}$, respectively. While this does imply that there will be fewer detected binaries containing mid-L companions than late-M companions, it does not imply the almost complete nondetection of the former that we observe.

To further test this theory, we modified the simulation from Paper I to randomly assign a distance from a distribution equivalent to that for our current targets, and then reject simulated binaries that would not be detected in 2MASS at the good level. We also tried a number of different distance distributions, such as increasing the relative number of targets at a given distance, $d$, in proportion to $d^{2}$ between several minimum and maximum distances, or assuming a strongly peaked population at a specific distance that falls off for larger and smaller distances. As suspected, 
although in all cases introducing some kind of distance dependence on the detectability of a simulated binary results in fewer late spectral type binaries in the simulated color-color diagram, there are still a substantial number of "detected" binaries with late-M to mid-L companions, which are not present in large numbers in the observed color-color diagram.

We can also explore the effect of the observed relative numbers of stars as a function of spectral type (or mass). By default, the simulation from Paper I (which was constructed purely to illustrate the possible loci of near-IR color-color space occupied by WD binaries) considers a binary with an M-type companion to be as likely as a binary with an L-type companion. In reality, however, only about $35 \%$ of the field stars within $20 \mathrm{pc}$ have spectral types of M5 or later, and only about $5 \%$ have spectral types of L0 or later; most ( $\approx 50 \%$ ) have spectral types of M3-4 (e.g., as compiled in Farihi et al. 2005a; see their Fig. 7). Taken at face value (however, see below), this suggests an explanation for why we do observe numerous candidate binaries with near-IR colors equivalent to main-sequence stars up to spectral types of M4-5. Considering the distribution of field-star spectral types, from our sample of 153 confirmed and candidate WD binaries we might expect 50-55 to contain dM5+ type companions, approximately eight of which are L-type companions. Approximately $50 \%$ of the red-excess WDs with known distances are closer than $d \approx 150$ pc (i.e., detectable if they contain a late-M companion); approximately $20 \%$ are closer than $d \approx 35$ pc (i.e., detectable if they contain a mid-L companion). So, to first order, we might still expect to detect 25-30 binaries with dM5+ companions, of which 1 or 2 have mid-L companions. The latter is roughly in agreement with our observed sample, if we count WD 0145-221 as the one detected WD + brown dwarf binary; however, the former is consistent only if we count essentially all of the binary candidates within $1 \sigma$ of the $\left(H-K_{S}\right)$ color of a dM5 star as binaries containing dM5+ companions.

Of course, the situation is more complex than this, since numerous recent surveys for WDs in binaries with low-mass companions have noted that there appears to be a mechanism that makes the existence of binaries containing a WD and very low mass (late-M through L type) companion less likely than that of a WD + mid-M or earlier companion, in excess of the relative numbers of low-mass field stars of these spectral types. The wellknown "brown dwarf desert" describes an observed dearth of solar-type stars (i.e., WD progenitors) in binaries with brown dwarf companions at separations $r \lesssim 5 \mathrm{AU}$, compared to the frequency of low-mass star + brown dwarf and brown dwarf + brown dwarf binaries (e.g., Marcy \& Butler 2000; Grether \& Lineweaver 2006). The brown dwarf desert might extend out to at least many hundreds of AU, although there is some evidence that the desert does not extend past separations of $r \gtrsim 1000 \mathrm{AU}$ (Gizis et al. 2001; however, also see the apparently contradictory results from Farihi et al. [2005a] discussed below, and McCarthy \& Zuckerman [2004]). An additional factor that could contribute to a lack of WDs with (close) brown dwarf companions is the possible destruction or outspiraling of preexisting low-mass companions during the post-main-sequence evolution of the WD progenitor (described in Paper III and references therein).

At distances of 5-400 pc, and assuming an angular resolution limit of $\sim 2^{\prime \prime}$, the binaries in our sample have likely separations of $r \lesssim 10-800$ AU. Farihi et al. (2005a) performed a search of 261 WDs sensitive to low-mass companions at separations of $r \sim 100-5000$ AU and an additional search of 86 WDs sensitive to low-mass companions at separations of $r \sim 50-1100$ AU. They detected no brown dwarf companions, implying that the fraction of WD + brown dwarf binaries, even at large separations, is
$<0.5 \%$ and does not reflect the larger relative populations of field stars of M-L spectral types (also see, e.g., Politano 2004; Dobbie et al. 2005). The Farihi et al. (2005a) fraction corresponds to $\lesssim 1$ WD + brown dwarf binary expected in our sample of 153 redexcess WDs, which is, again, consistent with the presence of WD 0145-221. Clearly, this result must be treated circumspectly because of the small number of expected, and observed, objects in this category, not to mention the fact that the likely range of sampled orbital separations is somewhat different between our survey and the Farihi surveys, which could produce an even smaller expected number of WD + brown dwarf binaries in our sample (because of the small numbers of red-excess WDs and very small expected fraction of WD + brown dwarf binaries, we did not attempt to quantify or correct for this effect). However, Farihi et al. (2005a, their Fig. 6) show that M5-9 stars as binary companions to WDs are found in only about $10 \%$ of the observed systems, compared to a frequency of about $30 \%$ in the field (however, as with the distribution of field stars, the peak spectral type for companions again occurs at M3-4). Thus, we should only expect $\approx 15$ WD binaries containing an M5-9 star, which is more consistent with the observed (small) number of candidates whose nominal $\left(H-K_{s}\right)$ color is redward of that of a dM5 star.

\section{CONCLUSIONS}

We have identified a large sample of candidates for binaries containing a WD and a cool main-sequence star of spectral type as late as approximately M4-5. However, we found only a small number of strong candidates for binaries containing companions of spectral types later than M5, and no new candidates for binaries containing an L type (i.e., potential brown dwarf) companion. This result is in agreement with the results from other recent searches for WD binaries, which suggest that the formation (and/or survival) rate for binaries with extreme mass ratios ${ }^{10}$ is very low.

An important result of our near-IR survey of WDs, begun in Paper I and culminating here, is simply the demonstration that the color selection method is a viable technique for selecting binary candidates and has a high confirmed success rate. The synthetic flux method (used in Paper III and Tremblay \& Bergeron 2007) is a logical next step, since it requires both additional data (e.g., multiple optical and IR bands) and the calculation of synthetic photometry from model atmospheres for each candidate star (e.g., it would have been impractical to attempt this type of analysis as a first step for the entire MS99 catalog). In the modern era of virtual observatories and all sky surveys (e.g., the upcoming all sky IR surveys to be performed by the Wide-field Infrared Survey Explorer and Akari/ASTRO-F), the color selection method that we have demonstrated using 2MASS photometry provides the most simple and efficient means to take advantage of existing, homogenous survey data in order to provide an initial list of binary candidates.

J. W. W. acknowledges financial support from a Harvey Mudd College National Merit Scholarship. M. G. acknowledges financial support from the Harvey S. Mudd Merit Award.

\footnotetext{
${ }^{10}$ That is, $M_{2} / M_{\text {pro }} \lesssim 0.05$, where $M_{2}$ is the initial mass of the companion and $M_{\text {pro }}$ is the mass of the WD progenitor. The initial companion mass could be smaller than the current companion mass due to material accreted by the companion during the post-main-sequence evolution of the WD progenitor (summarized in $\S 5.4$ of Farihi et al. 2005a). The progenitor mass, in turn, is likely a factor of $\gtrsim 2$ larger than the current WD mass (up to $\sim 10$ times larger for massive WDs; e.g., Weidemann 1987, 2000).
} 
Thanks to Tom Marsh (University of Warwick) for making his collection of WD finding charts available, and to Jessica Hall (University of Southern California) and Ryan Yamada (Harvey Mudd College) for their assistance in identifying white dwarfs. We also thank the anonymous referee for pointing out several papers that were helpful in improving the presentation of our results. This work was performed, in part, at the Jet Propulsion Laboratory (JPL), California Institute of Technology (CIT). Support for this work was provided by the National Aeronautics and Space Administration (NASA) under an Astrophysics Data Program grant issued through the Office of Space Science. This research made use of the NASA/Infrared Processing and Analysis Center (IPAC) Infrared Science Archive, which is operated by JPL/CIT, under contract with NASA, and data products from the Two Micron All Sky Survey, which is a joint project of the University of Massachusetts and IPAC/CIT, funded by NASA and the National Science Foundation (NSF). We used the SIMBAD database, operated at CDS, Strasbourg, France, and NASA's Astrophysics Data System. The National Geographic Society-Palomar Observatory Sky Atlas (POSS-I) was made by CIT with grants from the National Geographic Society. The Second Palomar Observatory Sky Survey (POSS-II) was made by CIT with funds from the NSF, the National Geographic Society, the Sloan Foundation, the Samuel Oschin Foundation, and the Eastman Kodak Corporation.

\section{APPENDIX}

\section{NOTES ON INDIVIDUAL OBJECTS}

WD 0018-267: Included (but not discussed) in Kilic et al. (2006); does not show mid-IR excess compared to model WD in their Figure 1.

WD 0023+388: Reference to binary status in MS99 is a private communication.

WD 0034-211: MS99 note "close double degenerate binary," but Bragaglia et al. (1990) reclassified it as a WD+dM.

WD 0041+092: BL Psc.

WD 0116-231: Eggen \& Bessell (1978) and Lamontagne et al. (2000) classify WD $0116-231$ as DA+dM (the latter may just be repeating the Eggen \& Bessell classification); however, Bessell \& Wickramasinghe (1979) list WD 0116-231 in their table of composite spectrum stars (dK or dM plus a blue star) as type sd0. Reference for binary status in MS99 is a preprint indicating the Montreal-Cambridge survey, with no obvious subsequent publication.

WD 0130-196: MS99 note "MCT 0130-1937 is a PG 1159 star with no detected variability."

WD J0148-255: WD 0145-257.

WD 0232+035: FS Cet, Feige 24. Orbital period of 4.232 days (Thorstensen et al. 1978; Ritter \& Kolb 2003).

WD J0254-053: WD 0252-055, HD 18131.

WD 0255+009.2: The 2 MASS colors do not strongly constrain a spectral type or luminosity class.

WD 0258+184: Possibly an sdB star, with an unresolved cool (G8) companion, misclassified as a WD in MS99 (Lisker et al. 2005).

WD 0302+621: Schmidt \& Smith (1995) find a marginal detection of an approximately kilogauss magnetic field, but no sign of a red companion in its optical spectrum (they were not specifically looking for binaries, but noted the possible presence of a red spectral component in several other WDs in their survey).

WD 0308+096: CC Cet. Orbital period of 0.287 days (Ritter \& Kolb 2003).
WD 0312+019: The 2MASS colors do not strongly constrain a spectral type.

WD J0357+286: WD 0353+284, V1092 Tau. Orbital period of 0.365 days (Jeffries et al. 1996). The WD may be accreting from the wind of its ultrafast rotating companion (Jeffries et al. 1996; Jeffries \& Smalley 1996).

WD 0413-077: 40 Eri $B$. The BC pair (A is a distant dK1 star) should be resolvable in 2MASS images, but the dM star is either undetected or unseen due to the brightness of the WD.

WD 0416+272: HL Tau 76, V411 Tau. A ZZ Ceti variable (MS99). Not identified as a binary in Farihi et al. (2005a).

WD 0419-487: RR Cae. Orbital period of 0.304 days (Ritter \& Kolb 2003).

WD 0429+176: HZ 9. Orbital period of 0.564 days (Ritter \& Kolb 2003).

WD 0458-662: Likely orbital period of $\sim 0.7$ days to several days (Hutchings et al. 1995).

WD 0627+299: Not identified as a binary in Farihi et al. (2005a).

WD 0628-020: Angular separation is near the 2MASS imaging resolution limit; however, no separate $2 \mathrm{MASS}$ data are available for the WD component, so the photometry results might be for the companion only.

WD 0710+741: HR Cam. Orbital period of 0.103 days (Ritter \& Kolb 2003).

WD 0718-316: IN CMa. Orbital period of 1.262 days (Ritter \& Kolb 2003).

WD 0800-533: Wickramasinghe \& Bessell (1977) suggest that this object is a possible cataclysmic variable; they do not show its spectrum, but say that $\mathrm{H} \alpha$ and $\mathrm{H} \beta$ have emission-line cores, and that it is a possible old nova. Red near-IR colors reported independently by Kawka et al. (2007).

WD 0802+387: Hot DZ star with strong $\mathrm{Ca}$ II $\mathrm{H}$ and $\mathrm{K}$ absorption lines (Sion et al. 1990). Not identified as a binary in Farihi et al. (2005a).

WD 0852+630: Reference for binary status in MS99 refers to wide $\left(a \approx 36^{\prime \prime}\right)$ common proper motion companion.

WD 0858-220: Reference for binary status in MS99 is a private communication. Barely resolved in 2MASS images; only one source in 2MASS PSC, at a position between the two stars, so the photometry could be for only the dM star or a blend of both.

WD 0908+226: Reference for binary status in MS99 is a private communication (no other literature citations).

WD 0915+201: Stellar image is slightly elongated (diameter $\approx 3^{\prime \prime}$ ) in red POSS images, and shows a distinct color gradient from blue at the northernmost end to red at the southernmost end in the combined POSS B+R+IR three-color image. The 2MASS source position is offset toward the red end, and there is no corresponding 2MASS source at the blue end, so it is possible that the 2MASS photometry corresponds only to the red star (i.e., the WD is undetected) or is a blend of both stars. The 2MASS colors do not strongly constrain a spectral type.

WD 0937-095: Reference for binary status in MS99 is a private communication, and probably refers to the $a \approx 13^{\prime \prime}$ common proper motion companion. There is some ambiguity in the literature about which star in the pair is the WD: 2MASS 09394969-0945562 is a closer match to the catalog coordinates of the WD (Salim \& Gould 2003) than the southern component (2MASS 09394977-0946098); however, both stars are about equally red in th near-IR (e.g., $J-K_{s} \approx 0.80-0.81$ ). We have used the northern component here, but the 2MASS photometry for the southern component was reported in Paper I. 
WD 0949+451: The red star is possibly a dM4.5+dM4.5 close binary separated by $0.009^{\prime \prime}$ (Paper III). Reference for binary status in MS99 is a preprint citing the Hamburg-Schmidt Catalog with no published follow-up.

WD 1013-050: Orbital period of 0.789 days (Ritter \& Kolb 2003).

WD 1026+002: UZ Sex. Orbital period of 0.597 days (Ritter \& Kolb 2003).

WD 1027-039: Reference for binary status in MS99 is a private communication, and probably refers to the wide $\left(a \approx 35^{\prime \prime}\right)$ common proper motion companion reported by Salim \& Gould (2003).

WD 1036-204: MS99 note " a polarized, carbon band, magnetic degenerate"; this might account for its near-IR colors instead of a red companion (see Paper II).

WD 1042-690: Orbital period of 0.337 days (Ritter \& Kolb 2003).

WD 1055-072: An unusual DC-type WD (Bergeron et al. 2001). No evidence for an unresolved red companion in the surveys of Debes et al. (2005b), Farihi et al. (2005a), and Kilic et al. (2006). Included (but not discussed) in Kilic et al. (2006); does not show mid-IR excess compared to model WD in their Figure 1.

WD 1101+364: MS99 note "double degenerate DA+DA double-lined" (also see Marsh 1995; Nelemans et al. 2005). Orbital period of 0.145 days (Ritter \& Kolb 2003).

WD 1104+044: Inspection of the POSS and 2MASS images shows that this is a not-quite-resolved $\left(a \approx 3^{\prime \prime}\right)$ common proper motion binary containing a very blue object (presumably the WD) and a red object; only the red object is detected by 2MASS.

WD 1106-211: Although it is the closest star to the correct coordinates, it is possible that this is not the WD because it does not display the large proper motion $\left(\mu=0.467^{\prime \prime} \mathrm{yr}^{-1}\right)$ reported by Evans (1992; however, no star within several arcminutes shows such proper motion).

WD 1126+185: Possibly an $\mathrm{sdB}+\mathrm{dG}-\mathrm{K}$ binary (Farihi et al. 2005a).

WD 1132-298: ESO 0439-095. This spectroscopic binary has a pair of wide $\left(a \approx 53^{\prime \prime}\right)$ common proper motion companions (ESO 0439-096A+B, which form an $a \approx 4^{\prime \prime}$ binary themselves) that are dM2 and dM3 stars, respectively.

WD 1136+667: Orbital period of 0.836 days (Ritter \& Kolb 2003).

WD 1156+129: This object has the correct optical brightness $(V \sim 17.5)$, position, and proper motion $\left(\mu=0.05^{\prime \prime} \mathrm{yr}^{-1}\right.$ at a position angle of $\theta=176^{\circ}$; Evans 1992), but is very red $(V-J \approx+2.8)$.

WD 1201+437: Xu et al. (1999) classify this object as a quasar; however, their X-ray position error circle has a $9^{\prime \prime}$ radius. The extremely red $2 \mathrm{MASS}$ colors of this object (e.g., $H-K_{s} \approx$ +1.1 ) lend credence to reclassifying it as a quasar.

WD 1213+528: EG UMa. Orbital period of 0.668 days (Ritter \& Kolb 2003).

WD 1224+309: LM Com. Orbital period of 0.259 days (Ritter \& Kolb 2003).

WD 1247-176: Orbital period of 0.571 days (Ritter \& Kolb 2003).

WD J1255+258: WD 1253+261, IN Com. Planetary nebula central star (PN G339.9+88.4). The hot $(T>100,000 \mathrm{~K}$; Feibelman \& Kaler 1983) component in IN Com is widely referred to as a subdwarf or WD precursor (e.g., Ritter 1986). It has been suggested in several literature sources that IN Com might be a triple system containing a close (possibly interacting) binary and a detached third component (likely a late $\mathrm{M}$ dwarf), although the identification of which stars are involved in the close versus wide binaries, and their corresponding orbital periods, has not been conclusively established (summarized in Strassmeier et al. 1997).

WD 1305+018: Stellar image is slightly elongated (diameter $\lesssim 2^{\prime \prime}$ ) in the east-west direction in the POSS and 2MASS images; the west end is slightly bluer than the east end, but there is no strong color gradient. Optical colors are redder than expected from its spectroscopic temperature, indicating a possible cool companion (Cheselka et al. 1993).

WD 1310-305: Not a close double WD binary (Maxted \& Marsh 1999).

WD 1314+293: HZ 43. Reference for binary status in MS99 is a private communication.

WD 1330+793: Reference for binary status in MS99 is a private communication. Only the red, eastern component of this common proper motion binary is detected by $2 \mathrm{MASS}$.

WD J1340+604: WD 1339+606.

WD 1412-109: Stellar image appears slightly elongated in POSS and 2MASS images; there is a slight color gradient in the combined POSS $\mathrm{B}+\mathrm{R}+\mathrm{IR}$ three-color image, with northwest end (faintly) red.

WD 1424+503: There is a red $(\mathrm{dMe})$ star $\approx 7.5^{\prime \prime}$ north of the WD (e.g., Mason et al. 1995) that is easily resolved in the POSS and 2MASS images (and the 2MASS PSC); it is not known whether this star is gravitationally bound with the WD. In addition, there is previous evidence for the WD having a close (unresolved) red companion (summarized in Schwartz et al. 1995).

WD 1433+538: Possibly a double degenerate (Liebert et al. 2005).

WD 1443+336: Possible cataclysmic variable (Green et al. 1986).

WD 1501+300: This is the object closest to the (imprecise) target coordinates listed in MS99, and is identified in the University of Arizona White Dwarf Database finding chart. However, there is a faint, blue star $\approx 72^{\prime \prime}$ west that might be the actual WD.

WD 1517+502: The companion in this binary is a dwarf carbon star; its 2 MASS colors mimic very late M to early L spectral type.

WD 1541-381: Reference for binary status in MS99 is a private communication, probably refers to the wide $\left(10.3^{\prime \prime}\right)$ common proper motion companion reported by Luyten (1949). This object is also reported as DA $+\mathrm{dM}$ (Bessell \& Wickramasinghe 1979), but this might also refer to the wide companion. The wide companion (2MASS 15451177-3818493) is slightly elongated in the northwest-southeast direction in the POSS (red) and 2MASS images and may itself be a close binary. The 2MASS photometry for both stars (the alleged WD and the wide companion) is equally red: $(J-H)=+0.52$ and $\left(H-K_{S}\right)=+0.24-0.32$.

WD 1558+616: Reference for binary status in MS99 is a preprint citing the Hamburg-Schmidt Catalog with no published follow-up.

WD 1603+125: The WD has an $8 \mathrm{MG}$ magnetic field (Wegner \& Swanson 1990a).

WD 1608+118: Stellar image is slightly elongated in the POSS images, with a strong color gradient in the combined POSS B+R+IR three-color image from red at the northwest end to blue at the southeast end. The 2MASS detection is offset toward the red end.

WD 1610+383: Inspection of POSS and 2MASS images shows that this is a common proper motion binary, barely resolved in the POSS images as a blue (southwest) and red (northeast) pair. The single 2MASS detection is located closer to the red star; see note in Paper I. There is also a much fainter 
star of intermediate (optical) color just north of the red component, but it does not appear to share proper motion with the binary.

WD 1619+525: Stellar image is slightly elongated in eastwest direction in (red) POSS images, with a color gradient in the combined POSS B+R+IR three-color image from blue (east) to red (west).

WD 1622+323: Finley et al. (1997) report an early M-type companion from an optical spectrum of this object.

WD 1631+781: Orbital period of 2.89 days (Ritter \& Kolb 2003).

WD 1632-227.1: Double degenerate (DC+DC) binary with separation of $a=5.75^{\prime \prime}$, resolved in all POSS and 2MASS images; component 1 is the northern, and somewhat brighter, of the two. We note that component 2 is even more red in the near-IR $\left(J-H \approx+0.7, H-K_{s} \approx+0.5\right)$, but is not included here because it is a moderate 2MASS All Sky detection $\left(\sigma_{K_{s}}>0.1 \mathrm{mag}\right)$. The 2MASS colors do not strongly constrain a spectral type.

WD 1634-573: MS99 note "Hot $(55,000 \mathrm{~K})$ DO degenerate with photospheric carbon in UV."

WD 1643+143: Optical photometry is too red for an isolated DA WD, suggesting an unresolved cool companion (Kidder et al. 1991).

WD 1654+160: V824 Her. Pulsating WD (Winget et al. 1984).

WD J1711+667: WD 1711+668. The 2MASS colors do not strongly constrain a spectral type.

WD 1717-345: The 2MASS colors do not strongly constrain a spectral type.

WD J1820+580: WD 1819+580.

WD 1845+019: Spectroscopic evidence for a long-period, red companion inferred from $\mathrm{H} \alpha$ emission line radial velocities (Maxted \& Marsh 1999). It is not known whether the close neighbor star described by Debes et al. (2005b) is gravitationally bound to the WD and whether it is the same spectroscopic companion star inferred by Maxted \& Marsh (1999). Schmidt \& Smith (1995) find no sign of a red companion in its optical spectrum (they were not specifically looking for binaries, but noted the possible presence of a red spectral component in several other WDs in their survey for magnetic fields).

WD 2009+622: Orbital period of 0.741 days (Ritter \& Kolb 2003).

WD J2013+400: WD 2011+395. Orbital period of 0.706 days (Ritter \& Kolb 2003).

WD J2024+200: WD 2022+198.
WD 2108-431: This object is the one indicated in the University of Arizona White Dwarf Database finding chart, but it is not blue in optical (POSS) images. There is a blue object $\approx 34^{\prime \prime}$ east, but that object is fuzzy and probably a galaxy.

WD 2131+066: IR Peg. Orbital period of 0.164 days (Ritter \& Kolb 2003). Pulsating (PG 1159 type) WD (MS99). The 2MASS colors do not strongly constrain a spectral type. Paunzen et al. (1998) suggest that the star observed at $0.3^{\prime \prime}$ separation is not the binary companion but, rather, the companion is an $\mathrm{M}$ dwarf in an even closer, as yet unresolved, orbit.

WD 2133+463: Common proper motion $\left(\mu=0.46^{\prime \prime} \mathrm{yr}^{-1}\right)$ binary with a separation of $a \approx 3^{\prime \prime}$ (Bakos et al. 2002). Possibly only the red component is detected by 2MASS (e.g., the stellar image does not appear elongated in 2MASS images).

WD 2154+408: Orbital period of 0.268 days (Ritter \& Kolb 2003; also see Hillwig et al. 2002).

WD 2237+819: Orbital period of 0.124 days (Ritter \& Kolb 2003). Reference for binary status in MS99 is a preprint indicating the Hamburg-Schmidt Survey (e.g., see Gänsicke et al. 2004).

WD 2256+249: MS Peg. Orbital period of 0.174 days (Ritter \& Kolb 2003).

WD 2311-068: Not identified as a binary in Farihi et al. (2005a). This is a cool DQ6-type WD (e.g., Dufour et al. 2005), which might account for its red near-IR colors.

WD 2318-137: This is a known common proper motion binary (MS99; Silvestri et al. 2002); there is a comoving blue object (a WD?) located $\approx 89^{\prime \prime}$ east of the red star whose 2 MASS designation is listed here; this is likely the companion noted in MS99 and Silvestri et al. (2002). However, the red star appears elongated in the southeast-to-northwest direction on the POSS (red) images, suggesting that it might also be a binary (with separation of $a \approx 3^{\prime \prime}$ ). The POSS blue and 2MASS images show the least elongation, suggesting that this closer binary has red and blue components (and possibly only the red component is detected by 2 MASS).

WD 2326-224: Stellar image is slightly elongated in all POSS images and the combined POSS B+R+IR three-color image shows a color gradient from blue (northwest) to red (southeast). The stellar image is not noticeably elongated in the 2MASS images, and the coordinates of the single 2MASS detection are offset toward the red end of the elongated optical image.

WD 2336-187: Believed to be a single WD (Tremblay \& Bergeron 2007).
Bakos, G. Á., Sahu, K. C., \& Németh, P. 2002, ApJS, 141, 187

Barstow, M. A., Bond, H. E., Burleigh, M. R., \& Holberg, J. B. 2001, MNRAS, 322,891

Becklin, E. E., Farihi, J., Jura, M., Song, I., Weinberger, A. J., \& Zuckerman, B. 2005, ApJ, 632, L119

Bergeron, P., Leggett, S. K., \& Ruiz, M. T. 2001, ApJS, 133, 413

Bessell, M. S., \& Brett, J. M. 1988, PASP, 100, 1134

Bessell, M. S., \& Wickramasinghe, D. T. 1979, ApJ, 227, 232

Bragaglia, A., Greggio, L., Renzini, A., \& D’Odorico, S. 1990, ApJ, 365, L13

Bruch, A., \& Diaz, M. P. 1998, AJ, 116, 908

Carpenter, J. M. 2001, AJ, 121, 2851

Cheselka, M., Holberg, J. B., Watkins, R., Collins, J., \& Tweedy, R. W. 1993, AJ, 106, 2365

Ciardullo, R., Bond, H. E., Sipior, M. S., Fullton, L. K., Zhang, C.-Y., \& Schaefer, K. G. 1999, AJ, 118, 488

Cooke, B. A., et al. 1992, Nature, 355, 61

Debes, J. H., Sigurdsson, S., \& Woodgate, B. E. 2005a, AJ, 130, 1221 2005b, ApJ, 633, 1168

Dobbie, P. D., Burleigh, M. R., Levan, A. J., Barstow, M. A., Napiwotzki, R., Holberg, J. B., Hubeny, I., \& Howell, S. B. 2005, MNRAS, 357, 1049

\section{REFERENCES}

Dufour, P., Bergeron, P., \& Fontaine, G. 2005, ApJ, 627, 404

Eggen, O. J., \& Bessell, M. S. 1978, ApJ, 226, 411

Eisenstein, D. J., et al. 2006, ApJS, 167, 40

Evans, D. W. 1992, MNRAS, 255, 521

Farihi, J., Becklin, E. E., \& Zuckerman, B. 2005a, ApJS, 161, 394

Farihi, J., \& Christopher, M. 2004, AJ, 128, 1868

Farihi, J., Hoard, D. W., \& Wachter, S. 2006, ApJ, 646, 480 (Paper III)

Farihi, J., Zuckerman, B., \& Becklin, E. E. 2005b, AJ, 130, 2237

Feibelman, W. A. 1986, PASP, 98, 1199

Feibelman, W. A., \& Kaler, J. B. 1983, ApJ, 269, 592

Finley, D. S., Koester, D., \& Basri, G. 1997, ApJ, 488, 375

Fleming, T. A., Green, R. F., Jannuzi, B. T., Liebert, J., Smith, P. S., \& Fink, H. 1993, AJ, 106, 1729

Gänsicke, B. T., Araujo-Betancor, S., Hagen, H.-J., Harlaftis, E. T., Kitsionas, S., Dreizler, S., \& Engels, D. 2004, A\&A, 418, 265

Giclas, H. L. 1958, Lowell Obs. Bull., 4, 1 1980, Lowell Obs. Bull., 8, 157

Gizis, J. E., Kirkpatrick, J. D., Burgasser, A., Reid, I. N., Monet, D. G., Liebert, J., \& Wilson, J. C. 2001, ApJ, 551, L163

Gizis, J. E., Monet, D. G., Reid, I. N., Kirkpatrick, J. D., Liebert, J., \& Williams, R. J. 2000, AJ, 120, 1085 
Graham, J. R., Matthews, K., Neugebauer, G., \& Soifer, B. T. 1990, ApJ, 357, 216

Green, P. J., Ali, B., \& Napiwotzki, R. 2000, ApJ, 540, 992

Green, R. F., Schmidt, M., \& Liebert, J. 1986, ApJS, 61, 305

Greenstein, J. L. 1975, ApJ, 196, L117

1976, ApJ, 207, L119

1984, ApJ, 276, 602

1986, AJ, 92, 867

Greenstein, J. L., \& Liebert, J. W. 1990, ApJ, 360, 662

Grether, D., \& Lineweaver, C. H. 2006, ApJ, 640, 1051

Hawley, S. L., et al. 2002, AJ, 123, 3409

Heber, U., Dreizler, S., \& Hagen, H.-J. 1996, A\&A, 311, L17

Hillwig, T. C., Gale, A. A., Honeycutt, R. K., \& Rengstorf, A. W. 2002, PASP, 114,756

Holberg, J. B., Oswalt, T. D., \& Sion, E. M. 2002, ApJ, 571, 512

Holberg, J. B., Sing, D., Barstow, M. A., \& Good, S. 2001, Astron. Ges. Meeting Abst., 18, 106

Hutchings, J. B., Crampton, D., Cowley, A. P., Schmidtke, P. C., McGrath, T. K., \& Chu, Y.-H. 1995, PASP, 107, 931

Jasniewicz, G., Thevenin, F., Monier, R., \& Skiff, B. A. 1996, A\&A, 307, 200

Jeffries, R. D., Burleigh, M. R., \& Robb, R. M. 1996, A\&A, 305, L45

Jeffries, R. D., \& Smalley, B. 1996, A\&A, 315, L19

Jura, M. 2003, ApJ, 584, L91

Kawka, A., Vennes, S., Schmidt, G. D., Wickramasinghe, D. T., \& Koch, R. 2007, ApJ, 654, 499

Kawka, A., Vennes, S., \& Thorstensen, J. R. 2004, AJ, 127, 1702

Kellett, B. J., et al. 1995, ApJ, 438, 364

Kidder, K. M., Holberg, J. B., \& Mason, P. A. 1991, AJ, 101, 579

Kilic, M., von Hippel, T., Mullally, F., Reach, W. T., Kuchner, M. J., Winget, D. E., \& Burrows, A. 2006, ApJ, 642, 1051

Kilkenny, D., O’Donoghue, D., Koen, C., Stobie, R. S., \& Chen, A. 1997, MNRAS, 287, 867

Kirkpatrick, J. D., et al. 2000, AJ, 120, 447

Koester, D., et al. 2001, A\&A, 378, 556

Lamontagne, R., Demers, S., Wesemael, F., Fontaine, G., \& Irwin, M. J. 2000, AJ, 119, 241

Lanning, H. H. 1982, ApJ, 253, 752

Lanning, H. H., \& Pesch, P. 1981, ApJ, 244, 280

Liebert, J., Bergeron, P., \& Holberg, J. B. 2005, ApJS, 156, 47

Liebert, J., Schmidt, G. D., Lesser, M., Stepanian, J. A., Lipovetsky, V. A., Chaffe, F. H., Foltz, C. B., \& Bergeron, P. 1994, ApJ, 421, 733

Lisker, T., Heber, U., Napiwotzki, R., Christlieb, N., Han, Z., Homeier, D., \& Reimers, D. 2005, A\&A, 430, 223

Luyten, W. J. 1949, ApJ, 109, 528

Luyten, W. J., \& Albers, H. 1979, LHS Atlas (Minneapolis: Univ. Minnesota)

Marcy, G. W., \& Butler, R. P. 2000, PASP, 112, 137

Margon, B., Liebert, J., Lampton, M., Spinrad, H., Bowyer, S., \& Gatewood, G. 1976, ApJ, 209, 525

Marsh, T. R. 1995, MNRAS, 275, L1

Mason, K. O., et al. 1995, MNRAS, 274, 1194

Maxted, P. F. L., \& Marsh, T. R. 1999, MNRAS, 307, 122

Maxted, P. F. L., O’Donoghue, D., Morales-Rueda, L., \& Napiwotzki, R. 2007, MNRAS, 376, 919

McCarthy, C., \& Zuckerman, B. 2004, AJ, 127, 2871

McCook, G. P., \& Sion, E. M. 1999, ApJS, 121, 1 (MS99)

Morales-Rueda, L., Marsh, T. R., Maxted, P. F. L., Nelemans, G., Karl, C., Napiwotzki, R., \& Moran, C. K. J. 2005, MNRAS, 359, 648

Mueller, B. E. A., \& Bues, I. 1987, Mitt. Astron. Ges. Hamburg, 70, 345

Nelemans, G., et al. 2005, A\&A, 440, 1087

Orosz, J. A., Wade, R. A., Harlow, J. J. B., Thorstensen, J. R., Taylor, C. J., \& Eracleous, M. 1999, AJ, 117, 1598

Oswalt, T. D., Hintzen, P. M., \& Luyten, W. J. 1988, ApJS, 66, 391
Parsons, S. B., Wray, J. D., Benedict, G. F., Henize, K. G., \& Laget, M. 1976, ApJ, 206, L71

Paunzen, E., Konig, M., \& Dreizler, S. 1998, A\&A, 331, 162

Politano, M. 2004, ApJ, 604, 817

Probst, R. G. 1983, ApJS, 53, 335

Putney, A. 1997, ApJS, 112, 527

Raymond, S. N., et al. 2003, AJ, 125, 2621

Reach, W. T., Kuchner, M. J., von Hippel, T., Burrows, A., Mullally, F., Kilic, M., \& Winget, D. E. 2005, ApJ, 635, L161

Reed, M. D., Kawaler, S. D., \& O’Brien, M. S. 2000, ApJ, 545, 429

Ritter, H. 1986, A\&A, 169, 139

Ritter, H., \& Kolb, U. 2003, A\&A, 404, 301

Ruiz, M. T., \& Maza, J. 1990, AJ, 99, 995

Saffer, R. A., Wade, R. A., Liebert, J., Green, R. F., Sion, E. M., Bechtold, J., Foss, D., \& Kidder, K. 1993, AJ, 105, 1945

Salim, S., \& Gould, A. 2003, ApJ, 582, 1011

Schmidt, G. D., \& Smith, P. S. 1995, ApJ, 448, 305

Schmidt, G. D., Smith, P. S., Harvey, D. A., \& Grauer, A. D. 1995, AJ, 110, 398

Schultz, G., Zuckerman, B., \& Becklin, E. E. 1996, ApJ, 460, 402

Schwartz, R. D., Dawkins, D., Findley, D., \& Chen, D. 1995, PASP, 107, 667

Silvestri, N. M., Oswalt, T. D., \& Hawley, S. L. 2002, AJ, 124, 1118

Silvestri, N. M., Oswalt, T. D., Wood, M. A., Smith, J. A., Reid, I. N., \& Sion, E. M. 2001, AJ, 121, 503

Silvestri, N. M., et al. 2006, AJ, 131, 1674

Sing, D. K., et al. 2004, AJ, 127, 2936

Sion, E. M., Holberg, J. B., Barstow, M. A., \& Kidder, K. M. 1995, PASP, 107, 232

Sion, E. M., Kenyon, S. J., \& Aannestad, P. A. 1990, ApJS, 72, 707

Skrutskie, M. F., et al. 2006, AJ, 131, 1163

Stepanian, J. A., Green, R. F., Foltz, C. B., Chaffee, F., Chavushyan, V. H., Lipovetsky, V. A., \& Erastova, L. K. 2001, AJ, 122, 3361

Strassmeier, K. G., Hubl, B., \& Rice, J. B. 1997, A\&A, 322, 511

Thorstensen, J. R., Charles, P. A., Bowyer, S., \& Margon, B. 1978, ApJ, 223, 260

Thorstensen, J. R., Vennes, S., \& Shambrook, A. 1994, AJ, 108, 1924

Tokunaga, A. T., Becklin, E. E., \& Zuckerman, B. 1990, ApJ, 358, L21

Tremblay, P.-E., \& Bergeron, P. 2007, ApJ, 657, 1013

van den Bos, W. H. 1926, Bull. Astron. Inst. Netherlands, 3, 128

Vennes, S., Mathioudakis, M., Doyle, J. G., Thorstensen, J. R., \& Byrne, P. B. 1995, A\&A, 299, L29

Vennes, S., \& Thorstensen, J. R. 1994, ApJ, 433, L29

Vennes, S., Thorstensen, J. R., \& Polomski, E. F. 1999, ApJ, 523, 386

Wachter, S., Hoard, D. W., Hansen, K. H., Wilcox, R. E., Taylor, H. M., \& Finkelstein, S. L. 2003, ApJ, 586, 1356 (Paper I)

Wegner, G. 1979, MNRAS, 187, 17

Wegner, G., \& McMahan, R. K. 1988, AJ, 96, 1933

Wegner, G., McMahan, R. K., \& Boley, F. I. 1987, AJ, 94, 1271

Wegner, G., \& Swanson, S. R. 1990a, AJ, 99, 330

. $1990 \mathrm{~b}, \mathrm{AJ}, 100,1274$

Weidemann, V. 1987, A\&A, 188, 74 . 2000, A\&A, 363, 647

Wellhouse, J. W., Hoard, D. W., Howell, S. B., Wachter, S., \& Esin, A. A. 2005, PASP, 117, 1378 (Paper II)

Wesemael, F., Green, R. F., \& Liebert, J. 1985, ApJS, 58, 379

Wickramasinghe, D. T., \& Bessell, M. S. 1977, MNRAS, 181, 713

Winget, D. E., Robinson, E. L., Nather, R. E., \& Balachandran, S. 1984, ApJ, 279, L15

Xu, D. W., Wei, J. Y., Dong, X. Y., \& Hu, J. Y. 1999, A\&AS, 134, 365

Zuckerman, B., \& Becklin, E. E. 1987, ApJ, 319, L99

Zuckerman, B., Koester, D., Reid, I. N., \& Hünsch, M. 2003, ApJ, 596, 477

Zwitter, T., \& Munari, U. 1995, A\&AS, 114, 575 